\title{
Quantum Hall ferromagnetic phases in the Landau level $N=0$ of a graphene bilayer
}

\author{
J. Lambert ${ }^{1}$ and R. Côté ${ }^{1}$ \\ ${ }^{1}$ Département de physique, Université de Sherbrooke, Sherbrooke, Québec, J1K 2R1, Canada
}

(Dated: July 19, 2018)

\begin{abstract}
In a Bernal-stacked graphene bilayer, an electronic state in Landau level $N=0$ is described by its guiding-center index $X$ (in the Landau gauge) and by its valley, spin, and orbital indices $\xi= \pm K, \sigma=$ \pm 1 , and $n=0,1$. When Coulomb interaction is taken into account, the chiral two-dimensional electron gas (C2DEG) in this system can support a variety of quantum Hall ferromagnetic (QHF) ground states where the spins and/or valley pseudospins and/or orbital pseudospins collectively align in space. In this work, we give a comprehensive account of the phase diagram of the C2DEG at integer filling factors $\nu \in[-3,3]$ in Landau level $N=0$ when an electrical potential difference $\Delta_{B}$ between the two layers is varied. We consider states with or without layer, spin, or orbital coherence. For each phase, we discuss the behavior of the transport gap as a function of $\Delta_{B}$, the spectrum of collective excitations and the optical absorption due to orbital pseudospin-wave modes. We also study the effect of an external in-plane electric field on a coherent state that has both valley and spin coherence and show that it is possible, in such a state, to control the spin polarization by varying the strength of the external in-plane electric field.
\end{abstract}

PACS numbers: 73.21.-b,73.22.Gk,72.80.Vp

\section{INTRODUCTION}

Electrons in a Bernal-stacked graphene bilayer 1,2 behave as a chiral two-dimensional gas of massive Dirac fermions $(\mathrm{C} 2 \mathrm{DEG})^{3}$. The chiral nature of the electrons lead to transport and optical properties that are different from those of conventional semiconductor 2DEG'S or of the 2DEG in monolayer graphene. In particular, in the absence of Coulomb interaction, the Landau level (LL) spectrum is given by $E_{N}= \pm \hbar \omega_{c}^{*} \sqrt{N(N+1)}$, where the Landau level index $N=0, \pm 1, \pm 2, \ldots$ and the effective cyclotron frequency $\omega_{c}^{*}=e B / m^{*} c$ where $B$ is the magnetic field and $m^{*}$ the effective mass of the electrons. Each Landau level is four-time degenerate when counting valley and spin degrees of freedom with the exception of Landau level $N=0$ which is eight-time degenerate. Indeed, an electronic state in Landau level $N=0$ is specified by its guiding-center $X$ (in the Landau gauge), spin $\sigma= \pm 1$, valley $\xi= \pm K$ and orbital $n=0,1$ indices ${ }^{1}$. (In Landau level $N=0$, valley and layer degrees of freedom are equivalent.) When the Coulomb interaction is negligible with respect to the disorder broadening at low temperature and when the small Zeeman splitting is neglected, the eight states in $N=0$ are degenerate and the Hall conductivity has plateaus at $\sigma_{x y}=4 M e^{2} / h$ where $M= \pm 1, \pm 2, \ldots \stackrel{4}{\text {. }}$.

In recent transport experiments $\mathrm{s}^{-12}$, it was shown that in sufficiently pure sample, when disorder is low or when the magnetic field is large enough, the Coulomb interaction completely lifts the degeneracy of the $N=0$ octet and lead to the formation of seven new plateaus in the Hall conductivity i.e. $\sigma_{x y}=\nu M e^{2} / h$, where $\nu \in[-3,3]$. These plateaus were attributed to the formation of broken-symmetry many-body ground states. These states can alternatively be described as quantum Hall ferromagnets (QHF's) where the spin and/or valley pseudospins and/or orbital pseudospins are sponta- neously and collectively aligned in space $\underline{\underline{13}}$.

In bilayer graphene, a top-bottom gates voltage imbalance can be applied to create a potential difference $\Delta_{B}$ (which we call the "bias" hereafter) between the two layers. In dual-gated bilayer graphene, $\Delta_{B}$ and the total density of electrons in the bilayer can be controlled independently. This allows the phase diagram of the C2DEG to be studied as a function of $\Delta_{B}$, magnetic field and temperature. Such study has been done by Weitz et al. .12 in high-quality bilayer graphene suspended between a top gate electrode and the substrate. The measurements show a series of phase transitions between different QHF states as $\Delta_{B}$ is increased at given filling factor and magnetic field. Special attention has been given to the filling factor $\nu=0$ where the precise nature of the ground state when $B \rightarrow 0$ near zero bias is still debated ${ }^{14}$. All experiments were done at relatively small magnetic field $B<10 \mathrm{~T}$ with the exception of the experiments reported in Ref. 9 where $B$ reached $35 \mathrm{~T}$.

Various aspects of the QHF states in bilayer graphene (in particular the nature and the evolution of the ground state of the C2DEG near charge neutrality as $B \rightarrow 0$ ) have been studied theoretically by a number of authors $15-23$. In the work of Gorbar et al. $\underline{\underline{17}}$ and Shizuya ${ }^{19}$, the phase diagram of the C2DEG as a function of bias for $\nu=0,1,2,3$ is presented. Gorbar et al. have considered the effect of both statio 17 and dynamical screenings $\frac{18}{18}$ of the Coulomb interaction. The modified gap equation captures the linear scaling of the transport gaps with the magnetic field which is seen in all the experiments at low magnetic field. The transport gaps are also strongly reduced with respect to the unscreened case and become more comparable to those observed experimentally. The dynamical screening was found to reproduce the offset in the behavior of the gap with magnetic field in the spin-polarized QHF state near zero bias which is seen in the experiments $\frac{10}{}$. Shizuya points out that the 
filled levels $N \leq-1$ cannot be considered as completely inert. Instead, they lead to a correction $\Lambda_{n}$ of the energy of the orbital levels $n=0,1$ that can change the ordering of these states in a way that depend on their occupation. A negative capacitance effect is also found that suppress rotation of the valley pseudospins.

The work that we present in this paper extends our previous study of the phase diagram of the C2DEG at zero bias $13,24-26$ and complements the study of Gorbar et al. and Shizuya. We give a comprehensive account of the phase diagram of the C2DEG in bilayer graphene at all integer filling factors $\nu \in[-3,3]$ in Landau level $N=0$ as a function of an applied bias $\Delta_{B}$ at a fixed magnetic field. Our analysis is based on an effective two-band model 27 which describes the low-energy physics near the valleys $K_{ \pm}$. We explicitly take into account as symmetry-breaking terms the Zeeman splitting $\Delta_{Z}$ and the bias $\Delta_{B}$. In the effective two-band model, the bias $\Delta_{B}$ lifts the degeneracy between the orbital levels $n=0$ and $n=1$ by a small amount $\xi \beta \Delta_{B}$ where $\beta=\gamma_{1} / \hbar \omega_{c}^{*}$ with $\gamma_{1}$ the interlayer hopping between carbon atoms that are part of a dimer. We also include in our model the interlayer next nearest-neighbor hopping term $\gamma_{4}$ between carbons atoms in the same sublattices. This term causes a small asymmetry in the electronic band structure and was neglected in previous studies $\underline{17}, \underline{19}$. It combines with the correction $\xi \beta \Delta_{B}$ to give an energy difference $\approx 2 \beta \gamma_{1} \gamma_{4} / \gamma_{0}+\xi \beta \Delta_{B}$ between the $n=1$ and $n=0$ orbital levels where $\gamma_{0}$ is the intralayer hopping energy between nearest-neighbors. This correction is thus finite at zero bias and breaks the orbital degeneracy. Our phase diagram is not electron-hole symmetric around $\nu=0$ and the sequence of phase transitions is different for each filling factor.

In our analysis, we treat the electron interaction in the Hartree-Fock approximation (HFA) and compute the collective excitations and electromagnetic absorption of the different phases of the C2DEG in the generalized random-phase approximation (GRPA). We include in our study both uniform and non-uniform states and allow for the possibility of any type of coherent (or QHF) state. By coherent state, we mean a state where the average value $\left\langle c_{\xi, \sigma, n, X}^{\dagger} c_{\xi^{\prime}, \sigma^{\prime}, n^{\prime}, X}\right\rangle \neq 0$ for $\xi \neq \xi^{\prime}$ and/or $\sigma \neq \sigma^{\prime}$ and/or $n \neq n^{\prime}$ where $c_{\xi, \sigma, n, X}^{\dagger}$ creates an electron in a state with quantum numbers $\xi, \sigma, n, X$. In our phase diagram which is summarized on Fig. 5, the layer-coherent states occur at very small bias because of the small interlayer distance $d=0.34 \mathrm{~nm}$ in bilayer graphene. As the bias is increased, we find around a critical bias corresponding to the regions where the Hall conductivity ceases to be quantized in the experiments $\frac{12}{2}$ a state with both layer and spin coherence. The orbital coherent states occur at a much larger bias corresponding to the situation where level $n=1$ gets lower in energy than level $n=0$ in valley $K_{-}$(see Fig. 4). In-between these coherent states are various incoherent states, some of which have been studied before $\frac{17}{}$. Interestingly, we find that the application of an electric field in the plane of the layers can produce a new state where all three coherences (layer, orbital and spin) are present. In such a state, it is possible to control the degree of spin polarization by changing the strength of the external in-plane electric field.

We also present a study the properties of the different ground states in the phase diagram. For all filling factors, we show how the transport gaps evolve with bias. In most cases, this evolution is qualitatively similar to that obtained with screening corrections $\frac{17}{}$. We compute all the intra-LL collective excitations in the various phases showing that all coherent states but the orbital state are characterized by a linearly-dispersing gapless (in the long-wavelength limit) Goldstone mode. This mode becomes gapped after the transition to an adjacent incoherent state. In the orbital phase, the orbital-pseudospin Goldstone mode dispersion is anisotropic and this mode becomes unstable at a finite wave vector indicating a transition to a charge-density-wave state ${ }^{25,26}$. We identify the number of spin-waves and orbital modes in each phase. These later modes are active in optical absorption. The inter-LL and some intra-LL magnetoexcitons have been computed recently $\underline{\underline{20}} \underline{\underline{23}}$ and we comment on the difference with our results and how the presence of the inter-LL magnetoexcitons in the spectrum may complicate the detection of the intra-LL excitations. The main results of our paper are summarized in Fig. 5 (phase diagram), Fig. 8 (transport gaps) and Fig. 9,10 (collective mode dispersions).

This paper is organized in the following way. Section II introduces the two-band model of bilayer graphene with the resulting LL spectrum in finite magnetic field. Section III summarizes the Hartree-Fock and generalized random-phase approximations that we use to take into account the Coulomb interaction and gives the formalism for the calculation of the electromagnetic absorption. Our numerical results for the phase diagram, transport gaps, collective excitations and optical absorption are presented in Sec. IV. In Sec. V, we show how the application of an in-plane electric field allow to control the spin polarization in some phases. We conclude in Sec. VI.

\section{TWO-BAND MODEL OF BILAYER GRAPHENE}

\section{A. Crystal structure and tight-binding Hamiltonian}

The crystal structure of a Bernal-stacked graphene bilayer is shown in Fig. 1 Each graphene layer is a twodimensional crystal with a honeycomb lattice structure. The honeycomb lattice can be described as a triangular Bravais lattice with a basis of two carbon atoms $A_{n}$ and $B_{n}$ where $n=1,2$ is the layer index. The two basis vectors are given by $\mathbf{a}_{1}=a_{0}(1 / 2,-\sqrt{3} / 2)$ and $\mathbf{a}_{2}=a_{0}(1,0)$, where $a_{0}=2.46 \AA=\sqrt{3} c$ is the lattice 
constant of the underlying triangular Bravais lattice and $c=1.42 \AA$ is the separation between two adjacent carbon atoms. The distance between the two graphene layers is $d=3.4 \AA$. In the Bernal stacking arrangement, the upper $A$ sublattice is directly on top of the lower $B$ sublattice while the upper $B$ sublattice is above the center of a hexagonal plaquette of the lower layer.

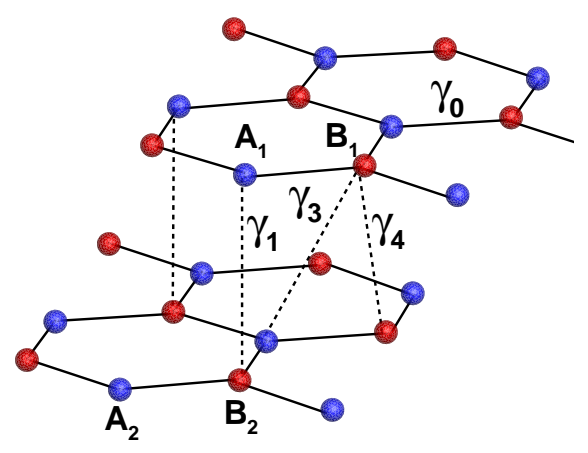

FIG. 1: (Color online) Crystal structure of a Bernal-stacked graphene bilayer.

The Brillouin zone of the reciprocal Bravais lattice is shown in Fig. 2, We choose the two nonequivalent valley points to be

$$
K_{\xi}=\left(\frac{2 \pi}{a_{0}}\right)\left(\xi \frac{2}{3}, 0\right),
$$

where $\xi= \pm$ is the valley index.

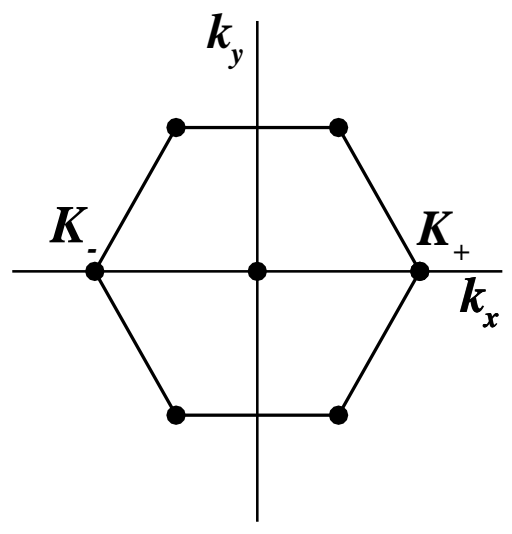

FIG. 2: Brillouin zone of the triangular Bravais lattice and definition of the valleys indices $K_{ \pm}$.

The electronic dispersion is obtained from a tightbinding model with the parameters ${ }^{1}$ : $\gamma_{0}$ the nearestneighbor $(\mathrm{NN})$ hopping in each layer, $\gamma_{1}$ the interlayer hopping between carbon atoms that are part of a dimer (i.e. $A_{1}-B_{2}$, these sites are called the high-energy sites), $\gamma_{3}$ the interlayer NN hopping term between carbon atoms of different sublattices (i.e. $A_{2}-B_{1}$ ) and $\gamma_{4}$ the interlayer next NN hopping term between carbons atoms in the same sublattice (i.e. $A_{1}-A_{2}$ and $B_{1}-B_{2}$ ). The energy $\delta$ represents the difference in the crystal field between sites $A_{1}, B_{2}$ and sites $A_{2}, B_{1}$. In this work, we neglect the trigonal warping term $\gamma_{3}$, a correct approximation at sufficiently high magnetic field ${ }^{27}$.

If we define the spinor

$$
d_{\mathbf{k}, \sigma}^{\dagger}=\left(\begin{array}{llll}
a_{1, \mathbf{k}, \sigma}^{\dagger} & b_{1, \mathbf{k}, \sigma}^{\dagger} & a_{2, \mathbf{k}, \sigma}^{\dagger} & b_{2, \mathbf{k}, \sigma}^{\dagger}
\end{array}\right)
$$

where $a_{i, \mathbf{k}, \sigma}^{\dagger}\left(b_{i, \mathbf{k}, \sigma}^{\dagger}\right)$ creates an electron on site $A(B)$ in layer $i=1,2$ with wave vector $\mathbf{k}$ and spin $\sigma= \pm 1$, then we can write the second-quantized tight-binding Hamiltonian in the basis $\left(A_{1}, B_{1}, A_{2}, B_{2}\right)$ as

$$
H^{0}=\sum_{\mathbf{k}, \sigma} d_{\mathbf{k}, \sigma}^{\dagger} H_{\sigma}^{0}(\mathbf{k}) d_{\mathbf{k}, \sigma}
$$

with the matrix

$$
\begin{aligned}
& H_{\sigma}^{0}(\mathbf{k})= \\
& \left(\begin{array}{cccc}
\frac{1}{2} \Delta_{B}+\delta & -\gamma_{0} \Lambda(\mathbf{k}) & -\gamma_{4} \Lambda^{*}(\mathbf{k}) & -\gamma_{1} \\
-\frac{1}{2} \sigma \Delta_{Z} & \frac{1}{2} \Delta_{B} & 0 & -\gamma_{4} \Lambda^{*}(\mathbf{k}) \\
-\gamma_{0} \Lambda^{*}(\mathbf{k}) & -\frac{1}{2} \sigma \Delta_{Z} & 0 & \\
-\gamma_{4} \Lambda(\mathbf{k}) & 0 & -\frac{1}{2} \Delta_{B} & -\gamma_{0} \Lambda(\mathbf{k}) \\
& & -\frac{1}{2} \sigma \Delta_{Z} & -\frac{1}{2} \Delta_{B}+\delta \\
-\gamma_{1} & -\gamma_{4} \Lambda(\mathbf{k}) & -\gamma_{0} \Lambda^{*}(\mathbf{k}) & -\frac{1}{2} \sigma \Delta_{Z}
\end{array}\right),
\end{aligned}
$$

where $\Delta_{Z}=g \mu_{B} B$ with $g=2$ is the Zeeman energy. We have also included in $H_{\sigma}^{0}$ an external transverse electric field that creates an electrical potential difference (or bias) $\Delta_{B}$ between the layers. The function $\Lambda(\mathbf{k})$ is defined by

$$
\Lambda(\mathbf{k})=\sum_{i=1}^{3} e^{i \mathbf{k} \cdot \delta_{i}}
$$

where the summation is over the vectors connecting a site $A_{1}$ to its three nearest-neighbors in the same plane i.e. $\delta_{1}=a_{0}(1 / 2,1 / 2 \sqrt{3}), \delta_{2}=a_{0}(-1 / 2,1 / 2 \sqrt{3}), \delta_{3}=$ $a_{0}(0,-1 / \sqrt{3})$.

Using $\Lambda\left(\mathbf{K}_{\xi}+\mathbf{k}\right) \approx-\sqrt{3} a_{0}\left(\xi k_{x}+i k_{y}\right) / 2$ and setting $\gamma_{4}=\delta=\Delta_{B}=\Delta_{Z}=0$, we find for the the band structure near the points $K_{\xi}$ the bands

$$
\begin{aligned}
& E_{1, \pm}(\mathbf{p})= \pm \gamma_{1} \pm \frac{p^{2}}{2 m^{*}} \\
& E_{2, \pm}(\mathbf{p})= \pm \frac{p^{2}}{2 m^{*}}
\end{aligned}
$$


where the momentum $\mathbf{p}$ is measured with respect to $\hbar \mathbf{K}_{\xi}$ and the effective mass is defined by

$$
m^{*}=\frac{2 \hbar^{2} \gamma_{1}}{3 \gamma_{0}^{2} a_{0}^{2}}
$$

The band structure consists of four bands. In the absence of bias, the two middle bands meet at the six valley points. The two high-energy bands are separated by a gap $\gamma_{1}$ from the two middle, low-energy bands. The bands $E_{2, \pm}(\mathbf{p})$ remain degenerate at $\mathbf{p}=0$ when $\gamma_{3}, \gamma_{4}$ and $\Delta$ are finite if $\Delta_{B}=0$. This degeneracy is lifted by a finite $\Delta_{B}$.

For a neutral bilayer, the chemical potential is at the energy $E=0$. The low-energy excitations $\left(E<<\gamma_{1}\right)$ of the tight-binding model can be studied using an effective two-band mode ${ }^{27,28}$. This model gives for each valley

$$
\begin{aligned}
& H_{\xi, \sigma}^{0}(\mathbf{p})= \\
& \left(\begin{array}{cc}
\xi \frac{\Delta_{B}}{2}+\eta_{-\xi} p_{-} p_{+} & \frac{1}{2 m^{*}}\left(p_{x}-i p_{y}\right)^{2} \\
-\frac{1}{2} \sigma \Delta_{Z} & -\xi \frac{\Delta_{B}}{2}+\eta_{\xi} p_{+} p_{-} \\
\frac{1}{2 m^{*}}\left(p_{x}+i p_{y}\right)^{2} & -\frac{1}{2} \sigma \Delta_{Z}
\end{array}\right),
\end{aligned}
$$

where we used the basis $\left(A_{2}, B_{1}\right)$ for $K_{-}$and $\left(B_{1}, A_{2}\right)$ for $K_{+}$and defined $p_{ \pm}=p_{x} \pm i p_{y}$ and

$$
\eta_{\xi}=\frac{1}{2 m^{*}}\left(\xi \frac{\Delta_{B}}{\gamma_{1}}+2 \frac{\gamma_{4}}{\gamma_{0}}+\frac{\delta}{\gamma_{1}}\right) .
$$

In this model, the presence of a quantizing perpendicular magnetic field is accounted for by making the Peierls substitution $\mathbf{p} \rightarrow \mathbf{P}=\mathbf{p}+e \mathbf{A} / c$ (with $e>0$ ), where $\nabla \times \mathbf{A}=\mathbf{B}=B \widehat{\mathbf{z}}$. Defining the ladder operators $a=\left(P_{x}-i P_{y}\right) \ell / \sqrt{2} \hbar$ and $a^{\dagger}=\left(P_{x}+i P_{y}\right) \ell / \sqrt{2} \hbar$ with the magnetic length $\ell=\sqrt{\hbar c / e B}$, we get

$$
H_{\xi, \sigma}^{0}=\left(\begin{array}{cc}
\xi \frac{\Delta_{B}}{2}+\zeta_{1,-} a a^{\dagger} & \zeta_{2} a^{2} \\
-\frac{1}{2} \sigma \Delta_{Z} & -\xi \frac{\Delta_{B}}{2}+\zeta_{1,+} a^{\dagger} a \\
\zeta_{2}\left(a^{\dagger}\right)^{2} & -\frac{1}{2} \sigma \Delta_{Z}
\end{array}\right)
$$

where

$$
\begin{aligned}
\zeta_{1} & =\beta\left(2 \frac{\gamma_{1} \gamma_{4}}{\gamma_{0}}+\delta\right), \\
\zeta_{1, \pm} & =\zeta_{1} \pm \xi \beta \Delta_{B} \\
\zeta_{2} & =\beta \gamma_{1}\left(1+2 \frac{\delta \gamma_{4}}{\gamma_{0} \gamma_{1}}+\left(\frac{\gamma_{4}}{\gamma_{0}}\right)^{2}\right),
\end{aligned}
$$

and

$$
\beta=\frac{\hbar \omega_{c}^{*}}{\gamma_{1}}
$$

The effective cyclotron frequency is $\omega_{c}^{*}=e B / m^{*} c$. In Eq. (11), the ladder operators are defined such that $a^{\dagger} \varphi_{n}(x)=i \sqrt{n+1} \varphi_{n+1}(x)$ and $a \varphi_{n}(x)=$ $-i \sqrt{n} \varphi_{n-1}(x)$ where $\varphi_{n}(x)$ with $n=0,1,2, \ldots$ are the eigenfunctions of the one-dimensional harmonic oscillator.

For all calculations done in this paper, we choose ${ }^{2}$ for the value of the parameters

$$
\begin{aligned}
\gamma_{0} & =3.1 \mathrm{eV} \\
\gamma_{1} & =0.39 \mathrm{eV} \\
\gamma_{4} & =0.12 \mathrm{eV} \\
\delta & =0.0156 \mathrm{eV} .
\end{aligned}
$$

We have checked that the band dispersion obtained with this choice of signs for the hopping terms is consistent with that reported in the literature ${ }^{29}$. With the magnetic field in Tesla, we have

$$
\begin{aligned}
\beta & =8.86 \times 10^{-3} B \\
\zeta_{1} & =0.404 B \mathrm{meV}
\end{aligned}
$$

while

$$
\begin{aligned}
\hbar \omega_{c}^{*} & =3.46 B \mathrm{meV} \\
\Delta_{Z} & =0.1158 B \mathrm{meV} \\
\alpha & =\frac{e^{2}}{\kappa \ell}=11.25 \sqrt{B} \mathrm{meV} .
\end{aligned}
$$

In the calculation of $\alpha$, we take $\kappa \approx 5$ for the effective dielectric constant at the position of the graphene layers. At $B=10 \mathrm{~T}$,

$$
\begin{aligned}
\zeta_{1} / \alpha & =3.5911 \times 10^{-2} \sqrt{B}=0.114 \\
\Delta_{Z} / \alpha & =1.0291 \times 10^{-2} \sqrt{B}=0.0325 \\
\hbar \omega_{c}^{*} / \alpha & =0.30756 \sqrt{B}=0.973 .
\end{aligned}
$$

\section{B. Landau levels and eigenstates of the non-interacting Hamiltonian}

When $\gamma_{4}=\delta=\Delta_{B}=\Delta_{Z}=0$, the Landau level spectrum of $H_{\xi, \sigma}^{0}$ is given by

$$
E_{N}^{0}=\operatorname{sgn}(N) \sqrt{|N|(|N|+1)} \hbar \omega_{c}^{*},
$$

where $N=0, \pm 1, \pm 2, \ldots$ is the Landau level index and sgn is the signum function. The corresponding eigenvectors of a given spin are

$$
\frac{1}{\sqrt{2}}\left(\begin{array}{c}
h_{|N|-1, X}(\mathbf{r}) \\
-\operatorname{sgn}(N) h_{|N|+1, X}(\mathbf{r})
\end{array}\right)
$$

for $N \neq 0$. We use the Landau gauge $\mathbf{A}=(0, B x, 0)$ where the eigenstates are

$$
h_{n, X}(\mathbf{r})=\frac{1}{\sqrt{L_{y}}} e^{-i X y / \ell^{2}} \varphi_{n}(x-X)
$$

with $X$ the guiding-center index. All Landau levels $N \neq 0$ are four-fold degenerate including spin and valley 
degrees of freedom in addition to the guiding-center degeneracy $N_{\varphi}=S / 2 \pi \ell^{2}$ where $S$ is the area of the 2DEG. The Landau level $N=0$ is an exception because there are two degenerate spinors with zero energy which are given, in the basis $\left(A_{2}, B_{1}\right)$ for $K_{-}$and $\left(B_{1}, A_{2}\right)$ for $K_{+}$, by

$$
\left(\begin{array}{c}
0 \\
h_{0, X}(\mathbf{r})
\end{array}\right),\left(\begin{array}{c}
0 \\
h_{1, X}(\mathbf{r})
\end{array}\right) .
$$

It follows that $N=0$ is eight-fold degenerate. In this paper, we restrict the Hilbert space to the Landau level $N=0$ and use the index $n=0,1$ to refer to the two "orbitals" $\varphi_{n=0}(x)$ and $\varphi_{n=1}(x)$. With finite values of $\gamma_{4}, \delta, \Delta_{Z}$ or $\Delta_{B}$, the valley, spin, and orbital degeneracies are lifted and the noninteracting energies become

$$
\begin{aligned}
& E_{\xi, \sigma, n=0}^{0}=-\frac{1}{2} \xi \Delta_{B}-\frac{1}{2} \sigma \Delta_{Z} \\
& E_{\xi, \sigma, n=1}^{0}=-\frac{1}{2} \xi \Delta_{B}-\frac{1}{2} \sigma \Delta_{Z}+\xi \beta \Delta_{B}+\zeta_{1} .
\end{aligned}
$$

The corresponding eigenspinors are still given by Eq. (31). Note that the structure of the sublattice spinors in Eqs. (31) is such that states from different valleys (which are localized on different layers) have no overlap. For $N=0$, the layer index is thus equivalent to the valley index.

\section{Limit of validity of the two-band model}

Fig. 3 shows a comparison between the four-band and two-band models for the electronic dispersion in Landau levels $N=-2,-1,0,1,2$ and valley $K_{-}$using the values of the parameters given previously. The agreement between the two models is excellent for $N=0$ where the difference in energy is of the order of $1 \%$. For levels $|N|>0$, the difference in energy between the two models is much more important. Note that the two sub-Landau levels of $N=0$ intersect level $N=1$ at $\Delta_{B} \approx 0.15 \mathrm{eV}$. For the valley $K_{+}$(not shown in the figure) the crossing occurs at a smaller bias $\Delta_{B} \approx 0.10 \mathrm{eV}$ corresponding to an electric field $E_{\perp} \approx 300 \mathrm{meV} / \mathrm{nm}$ between the layers. In our calculation we must keep the bias smaller than $\approx 0.10 \mathrm{eV}$ (i.e. $\Delta_{B} / \alpha \lesssim 2.8$ for $\kappa=5$ ) for our model to be valid.

Fig. 4 shows the ordering of the four levels of a given spin in $N=0$ at finite bias. The correction $\zeta_{1}$ opens a gap between the two orbital states $n=0$ and $n=1$ which is independent of the bias. The effective two-band model introduces a correction $\beta \Delta_{B}$ to this gap that has different signs in the two valleys as indicated in the figure. When combined with $\zeta_{1}$, the gap in valley $K_{+}$is positive at all biases while the gap in valley $K_{-}$changes sign (level $n=1$ gets below level $n=0$ ) when $\beta \Delta_{B}>\zeta_{1}$ i.e. for $\Delta_{B}>0.046 \mathrm{eV}$ (i.e. $\Delta_{B} / \alpha=1.3$ for $\kappa=5$ ).

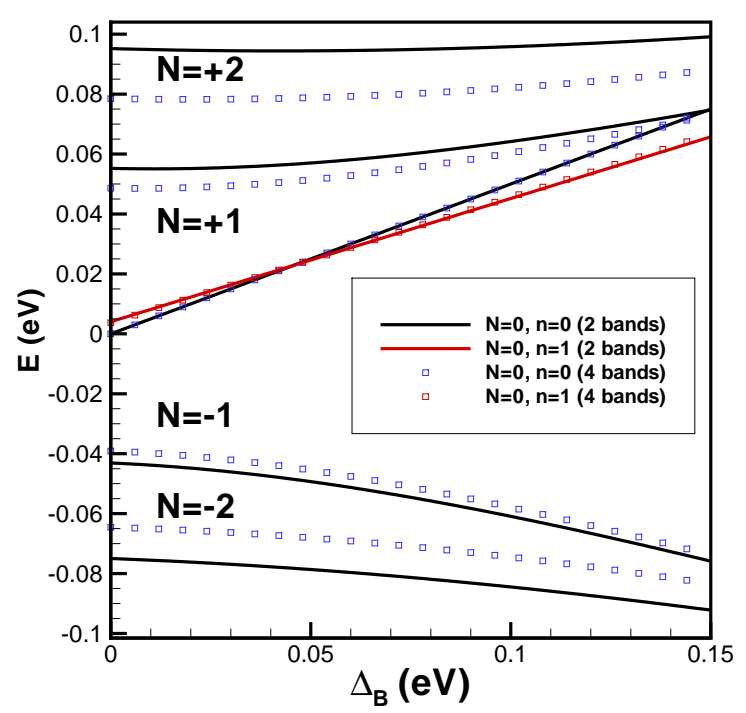

FIG. 3: (Color online) Comparison between the complete four-band model (symbols) and the approximate two-band model (lines) for the electronic dispersion in Landau levels $N=-2,-1,0,1,2$ and valley $K_{-}$.

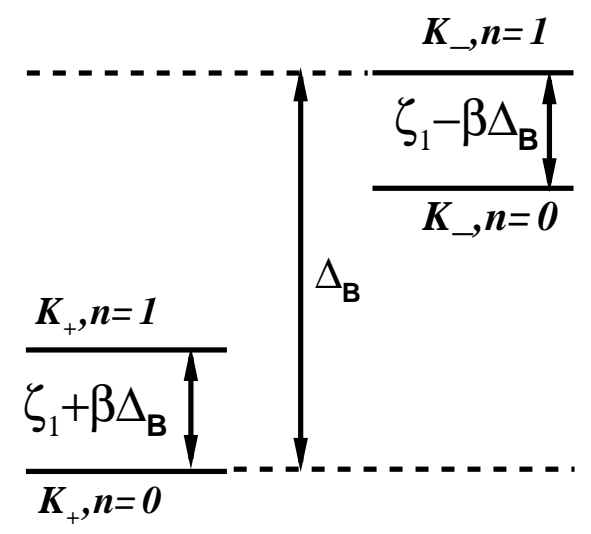

FIG. 4: Ordering of the four levels of a given spin in Landau level $N=0$ at finite bias $\Delta_{B}$.

\section{INTERACTING CHIRAL TWO-DIMENSIONAL ELECTRON GAS}

We now add the Coulomb interaction to the noninteracting Hamiltonian. Hereafter, we use the same basis $\left(A_{2}, B_{1}\right)$ for both valleys and define the field operators $\Psi_{\xi, \sigma, n}(\mathbf{r})$ by

$$
\Psi_{-, \sigma, n}(\mathbf{r})=\sum_{X}\left(\begin{array}{c}
0 \\
h_{n, X}(\mathbf{r})
\end{array}\right) \otimes|\sigma\rangle c_{-, \sigma, n, X}
$$


and

$$
\Psi_{+, \sigma, n}(\mathbf{r})=\sum_{X}\left(\begin{array}{c}
h_{n, X}(\mathbf{r}) \\
0
\end{array}\right) \otimes|\sigma\rangle c_{+, \sigma, n, X} .
$$

The second-quantized noninteracting part of the Hamiltonian is given by

$$
\begin{aligned}
H_{0} & =\sum_{\sigma, \xi, n} \int d \mathbf{r} \Psi_{\xi, \sigma, n}^{\dagger}(\mathbf{r}) H_{\xi, \sigma}^{0} \Psi_{\xi, \sigma, n}(\mathbf{r}) \\
& =\sum_{\xi, \sigma, n} \sum_{X} E_{\xi, \sigma, n}^{0} c_{\xi, \sigma, n, X}^{\dagger} c_{\xi, \sigma, n, X} .
\end{aligned}
$$

For the second-quantized Coulomb interaction,

$$
\begin{aligned}
V= & \frac{1}{2} \sum_{n_{1}, \ldots, n_{4}} \sum_{\sigma, \sigma^{\prime}} \sum_{\xi, \xi^{\prime}} \int d \mathbf{r} \int d \mathbf{r}^{\prime} \Psi_{\xi, \sigma, n_{1}}^{\dagger}(\mathbf{r}) \\
& \times \Psi_{\xi^{\prime}, \sigma^{\prime}, n_{2}}^{\dagger}\left(\mathbf{r}^{\prime}\right) V_{\xi, \xi^{\prime}}\left(\mathbf{r}-\mathbf{r}^{\prime}\right) \Psi_{\xi^{\prime}, \sigma^{\prime}, n_{3}}\left(\mathbf{r}^{\prime}\right) \Psi_{\xi, \sigma, n_{4}}(\mathbf{r}),
\end{aligned}
$$

where the Coulomb potential

$$
V_{\xi, \xi^{\prime}}(\mathbf{r})=\frac{e^{2}}{\kappa\left|\mathbf{r}-\mathbf{r}^{\prime}+\left(1-\delta_{\xi, \xi^{\prime}}\right) d \widehat{\mathbf{z}}\right|}
$$

has the Fourier transform

$$
V_{\xi, \xi^{\prime}}(\mathbf{r})=\frac{1}{S} \sum_{\mathbf{q}} \frac{2 \pi e^{2}}{\kappa q} e^{i \mathbf{q} \cdot\left(\mathbf{r}-\mathbf{r}^{\prime}\right)} e^{-q d\left(1-\delta_{\xi, \xi^{\prime}}\right)},
$$

where $\mathbf{q}$ is a two-dimensional vector in the plane of the bilayer. The terms that do not conserve the valley index in Eq. (37) are very small and usually neglected ${ }^{30}$.

\section{A. Hartree-Fock Hamiltonian}

In order to describe the different phases of the uniform C2DEG, we define the operators

$$
\rho_{n, n^{\prime}}^{a, a^{\prime}}=\frac{1}{N_{\varphi}} \sum_{X} c_{a, n, X}^{\dagger} c_{a^{\prime}, n^{\prime}, X}
$$

where $c_{a, n, X}^{\dagger}\left(c_{a, n, X}\right)$ creates(destroys) an electron in a state $(a, n, X)$. The index $a$ combines the spin and valley indices and we use $a_{\xi}$ and $a_{\sigma}$ to refer to the specific spin or valley index of $a=\left(a_{\xi}, a_{\sigma}\right)$. The set of average values $\left\{\left\langle\rho_{n, n^{\prime}}^{a, a^{\prime}}\right\rangle\right\}$ gives a complete description of an uniform ground state. They are the order parameters of that state. The diagonal elements $\left\{\left\langle\rho_{n, n}^{a, a}\right\rangle\right\}$ are the filling factors of levels $(a, n)$, while the off-diagonal elements are the "coherences". For nonuniform states, it is necessary to define the order parameters $\left\{\left\langle\rho_{n, n^{\prime}}^{a, a^{\prime}}(\mathbf{G})\right\rangle\right\}$ where $\mathbf{G}$ is a reciprocal lattice vector and $\left\langle\rho_{n, n^{\prime}}^{a, a^{\prime}}(\mathbf{G})\right\rangle$ the Fourier transform of $\left\langle\rho_{n, n^{\prime}}^{a, a^{\prime}}(\mathbf{r})\right\rangle$. We refer the reader to Refs. 25, 26 where the formalism for this case is discussed in more details. The Hartree-Fock Hamiltonian can be written in terms of these operators by (we adopt the convention that repeated indices are summed over)

$$
\begin{gathered}
H_{H F}=N_{\varphi} E_{a, n} \rho_{n, n}^{a, a} \\
-N_{\varphi} X_{n_{1}, n_{4}, n_{3}, n_{2}}^{\left(a_{\xi}, b_{\xi}\right)}(0)\left\langle\rho_{n_{1}, n_{2}}^{a, b}\right\rangle \rho_{n_{3}, n_{4}}^{b, a},
\end{gathered}
$$

where

$$
E_{a, n}=E_{a, n}^{0}+\alpha\left(\widetilde{\nu}_{a_{\xi}}-\frac{\widetilde{\nu}}{2}\right) \frac{d}{\ell},
$$

with $E_{a, n}^{0}$ given by Eqs. (32, 33). The Fock interaction is defined by

$$
\begin{aligned}
X_{n_{1}, n_{2}, n_{3}, n_{4}}^{\left(a_{\xi}, b_{\xi}\right)}(\mathbf{q})= & \alpha \int \frac{d \mathbf{p} \ell^{2}}{2 \pi} \frac{1}{p \ell} K_{n_{1}, n_{2}}(\mathbf{p}) \\
& \times K_{n_{3}, n_{4}}(-\mathbf{p}) e^{i \mathbf{q} \times \mathbf{p} \ell^{2}} e^{-p d\left(1-\delta_{a_{\xi}, b_{\xi}}\right)}
\end{aligned}
$$

with the form factors

$$
\begin{aligned}
& K_{0,0}(\mathbf{q})=e^{-\frac{q^{2} \ell^{2}}{4}}, \\
& K_{1,1}(\mathbf{q})=e^{-\frac{q^{2} \ell^{2}}{4}}\left(1-\frac{q^{2} \ell^{2}}{2}\right), \\
& K_{1,0}(\mathbf{q})=e^{-\frac{q^{2} \ell^{2}}{4}}\left(\frac{\left(q_{y}+i q_{x}\right) \ell}{\sqrt{2}}\right), \\
& K_{0,1}(\mathbf{q})=e^{-\frac{q^{2} \ell^{2}}{4}}\left(\frac{\left(-q_{y}+i q_{x}\right) \ell}{\sqrt{2}}\right) .
\end{aligned}
$$

These form factors capture the character of the two different orbital states. In Eq. (42), $\widetilde{\nu}=\nu+4$ is the number of filled levels in $N=0$. We reserve the symbol $\nu \in[-3,3]$ for the filling factor of the C2DEG. In deriving Eq. (41), we have taken into account a neutralizing positive background so that the only contribution from the Hartree and background terms is the capacitive energy given by the term in parenthesis in Eq. (42). In this term, $\widetilde{\nu}_{a_{\xi}}=\sum_{n, \sigma}\left\langle\rho_{n, n}^{a_{\xi}, \sigma ; a_{\xi}, \sigma}(0)\right\rangle$ is the total filling factor in valley $a_{\xi}$. Detailed expressions for the Hartree (see next section) and Fock interactions $H$ and $X$ are given in Appendix A of Ref. 25.

The Hartree-Fock energy per electron is given by

$$
\begin{gathered}
\frac{E_{H F}}{N_{e}}=\frac{1}{\widetilde{\nu}} E_{a, n}^{0}\left\langle\rho_{n, n}^{a, a}\right\rangle \\
+\frac{1}{4 \widetilde{\nu}} \frac{d}{\ell} \alpha\left(\widetilde{\nu}_{K_{+}}-\widetilde{\nu}_{K_{-}}\right)^{2} \\
-\frac{1}{2 \widetilde{\nu}} X_{n_{1}, n_{4}, n_{3}, n_{2}}^{\left(a_{\xi}, b_{\xi}\right)}(0)\left\langle\rho_{n_{1}, n_{2}}^{a, b}\right\rangle\left\langle\rho_{n_{3}, n_{4}}^{b, a}\right\rangle,
\end{gathered}
$$

where $N_{e}$ is the number of electrons in the 2DEG and $\nu_{K_{ \pm}}$are the filling factors of the two valleys.

At $\mathbf{q}=0$, the only nonzero matrix elements of the Fock interactions are

$$
\begin{aligned}
& X_{0,0,0,0}^{\xi, \xi}(0)=\Delta_{C}, X_{1,1,1,1}^{\xi, \xi}(0)=\frac{3}{4} \Delta_{C}, \\
& X_{0,0,1,1}^{\xi, \xi}(0)=X_{1,1,0,0}^{\xi, \xi}(0)=\frac{1}{2} \Delta_{C}, \\
& X_{1,0,0,1}^{\xi, \xi}(0)=X_{0,1,1,0}^{\xi, \xi}(0)=\frac{1}{2} \Delta_{C},
\end{aligned}
$$


and the corresponding interlayer terms which must be computed numerically. We have defined

$$
\Delta_{C}=\sqrt{\frac{\pi}{2}} \alpha
$$

with $\alpha=e^{2} / \kappa \ell$.

\section{B. Calculation of the order parameters} tion

We define the single-particle Matsubara Green's func-

$$
G_{n_{1, n_{2}}}^{a, b}(X, \tau)=-\left\langle T_{\tau} c_{a, n_{1}, X}(\tau) c_{b, n_{2}, X}^{\dagger}(0)\right\rangle
$$

where $T_{\tau}$ is the imaginary time ordering operator, such that the order parameters are given by

$$
\left\langle\rho_{n_{1}, n_{2}}^{a, b}\right\rangle=\frac{1}{N_{\varphi}} \sum_{X} G_{n_{2}, n_{1}}^{b, a}\left(X, \tau=0^{-}\right) .
$$

The equation of motion for the Green's function in the Hartree-Fock approximation is

$$
\begin{gathered}
\left(i \hbar \omega_{n}+\mu-E_{a, n_{1}}\right) G_{n_{1}, n_{2}}^{a, b}\left(i \omega_{n}\right) \\
+U_{n_{1}, n_{3}}^{a, c} G_{n_{3}, n_{2}}^{c, b}\left(i \omega_{n}\right)=\hbar \delta_{n_{1}, n_{2}} \delta_{a, b},
\end{gathered}
$$

where $\mu$ is the chemical potential, $\omega_{n}$ a fermionic Matsubara frequency and

$$
U_{n_{1}, n_{3}}^{a, c}=X_{n_{4}, n_{3}, n_{1}, n_{2}}^{\left(a_{\xi}, c_{\xi}\right)}(0)\left\langle\rho_{n_{4}, n_{2}}^{c, a}\right\rangle
$$

are the self-consistent Fock potentials.

The self-consistent Eq. (55) can be put in a $8 \times 8$ matrix form by defining superindices and then solved numerically in an iterative way in order to get the order parameters.

The Hartree-Fock equation of motion for the Green's function leads to the sum rule (at $T=0 \mathrm{~K}$ )

$$
\sum_{b, m}\left|\left\langle\rho_{n, m}^{a, b}\right\rangle\right|^{2}=\left\langle\rho_{n, n}^{a, a}\right\rangle=\nu_{n}^{a}
$$

where $\nu_{n}^{a}$ is the filling factor of the $(a, n)$ level. By definition

$$
\left\langle\rho_{n, m}^{a, b}\right\rangle=\left\langle\rho_{m, n}^{b, a}\right\rangle^{*}
$$

\section{Collective modes in the generalized random-phase approximation}

To study the collective excitations, we compute the two-particle Green's functions

$$
\begin{aligned}
& \chi_{n_{1}, n_{2}, n_{3}, n_{4}}^{a, b, c, d}(\mathbf{q}, \tau) \\
& =-N_{\varphi}\left\langle T_{\tau} \rho_{n_{1}, n_{2}}^{a, b}(\mathbf{q}, \tau) \rho_{n_{3}, n_{4}}^{c, d}(-\mathbf{q}, 0)\right\rangle \\
& +N_{\varphi}\left\langle\rho_{n_{1}, n_{2}}^{a, b}(\mathbf{q})\right\rangle\left\langle\rho_{n_{3}, n_{4}}^{c, d}(-\mathbf{q})\right\rangle
\end{aligned}
$$

in the generalized random-phase approximation (GRPA). In this approximation, $\chi_{n_{1}, n_{2}, n_{3}, n_{4}}^{a, b, c, d}(\mathbf{q}, \tau)$ is the solution of the equation

$$
\begin{aligned}
& \chi_{n_{1}, n_{2}, n_{3}, n_{4}}^{a, b, c, d}\left(\mathbf{q}, i \Omega_{n}\right) \\
& =\chi_{n_{1}, n_{2}, n_{3}, n_{4}}^{(0) a, b, c, d}\left(\mathbf{q}, i \Omega_{n}\right) \\
& +\frac{1}{\hbar} \chi_{n_{1}, n_{2}, n_{5}, n_{6}}^{(0) a, b, e,}\left(\mathbf{q}, i \Omega_{n}\right) \\
& \times H_{n_{5}, n_{6}, n_{7}, n_{8}}^{\left(e_{\xi}, g_{\xi}\right)}(\mathbf{q}) \chi_{n_{7}, n_{8}, n_{3}, n_{4}}^{g, g, c, d}\left(\mathbf{q}, i \Omega_{n}\right) \\
& -\frac{1}{\hbar} \chi_{n_{1}, n_{2}, n_{5}, n_{6}}^{(0) a, b, e, f}\left(\mathbf{q}, i \Omega_{n}\right) \\
& \times X_{n_{5}, n_{8}, n_{7}, n_{6}}^{\left(e_{\xi}, f_{\xi}\right)}(\mathbf{q}) \chi_{n_{7}, n_{8}, n_{3}, n_{4}}^{f, e, c, d}\left(\mathbf{q}, i \Omega_{n}\right) \text {, }
\end{aligned}
$$

where $\Omega_{n}$ is a bosonic Matsubura frequency and the Hartree interaction

$$
\begin{aligned}
H_{n_{1}, n_{2}, n_{3}, n_{4}}^{\left(a_{\xi}, b_{\xi}\right)}(\mathbf{q})= & \frac{1}{q \ell} K_{n_{1}, n_{2}}(\mathbf{q}) K_{n_{3}, n_{4}}(-\mathbf{q}) \\
& \times e^{-q d\left(1-\delta_{a_{\xi}, b_{\xi}}\right)}
\end{aligned}
$$

The two-particle Green's functions $\chi_{n_{1}, n_{2}, n_{3}, n_{4}}^{(0) a, b, c, i}\left(\mathbf{q}, i \Omega_{n}\right)$ satisfy the set of equations

$$
\begin{aligned}
& {\left[i \hbar \Omega_{n}-\left(E_{b, n_{2}}-E_{a, n_{1}}\right)\right] \chi_{n_{1}, n_{2}, n_{3}, n_{4}}^{(0) a, b, c, d}\left(\mathbf{q}, \Omega_{n}\right) } \\
= & \hbar\left\langle\rho_{n_{1}, n_{4}}^{a, d}\right\rangle \delta_{b, c} \delta_{n_{2}, n_{3}}-\hbar\left\langle\rho_{n_{3}, n_{2}}^{c, b}\right\rangle \delta_{a, d} \delta_{n_{1}, n_{4}} \\
& +U_{m, n_{1}}^{a, e} \chi_{m, n_{2}, n_{3}, n_{4}}^{(0) e, b, c, d}\left(\mathbf{q}, \Omega_{n}\right) \\
& -U_{n_{2}, m}^{e, b} \chi_{n_{1}, m, n_{3}, n_{4}}^{(0) a, e, c, d}\left(\mathbf{q}, \Omega_{n}\right)
\end{aligned}
$$

Eq. (60) can be represented by a set of bubbles (Hartree terms) and ladder (Fock terms) diagrams. The function $\chi_{n_{1}, n_{2}, n_{3}, n_{4}}^{(0) a, b}$ is the Hartree-Fock approximations for the two-particle Green's functions. It includes the HartreeFock self-energy corrections but not the vertex corrections. Note that two-particle Green's functions depend only on the order parameters $\left\langle\rho_{n, m}^{a, b}\right\rangle$ computed in the HFA. Eqs. 6062) can be solved numerically by defining superindices and then writing them in a $64 \times 64$ matrix form. The collective excitations are then given by the poles of the retarded Green's functions $\chi_{n_{1}, n_{2}, n_{3}, n_{4}}^{(R) a, b, d}(\mathbf{q}, \omega)$ which are obtained by the analytic continuation $i \Omega_{n} \rightarrow$ $\omega+i \delta$ of the corresponding two-particle Green's functions. To derive the dispersion relations, we follow these poles as the wave vector $\mathbf{q}$ is varied.

\section{Pseudospin description}

We showed above that the coherent states of the C2DEG can be described by the set of order parameters $\left\{\left\langle\rho_{n, n^{\prime}}^{a, a^{\prime}}\right\rangle\right\}$. These states are also quantum Hall ferromagnets (QHF's) and can also be described by using a pseudospin language where the two valley states $\xi=+$ $(\xi=-)$ are associated with valley-pseudospin up (down) 
and the two orbital states $n=0(n=1)$ with orbitalpseudospin up (down).

In this language, the total spin, valley pseudospin, and orbital pseudospin components of the electron gas are given by

$$
\begin{aligned}
S_{i} & =\frac{1}{2 N_{\varphi}} \hbar \sum_{\xi, n, X} \sum_{\alpha, \beta}\left\langle c_{\xi, \alpha, n, X}^{\dagger} \sigma_{\alpha, \beta}^{(i)} c_{\xi, \beta, n, X}\right\rangle, \\
L_{i} & =\frac{1}{2 N_{\varphi}} \sum_{\alpha, n, X} \sum_{\xi, \xi^{\prime}}\left\langle c_{\xi, \alpha, n, X}^{\dagger} \sigma_{\xi, \xi^{\prime}}^{(i)} c_{\xi^{\prime}, \alpha, n, X}\right\rangle, \\
O_{i} & =\frac{1}{2 N_{\varphi}} \sum_{\xi, \alpha, X} \sum_{n, n^{\prime}}\left\langle c_{\xi, \alpha, n, X}^{\dagger} \sigma_{n, n^{\prime}}^{(i)} c_{\xi, \alpha, n^{\prime}, X}\right\rangle,
\end{aligned}
$$

where $\sigma^{(i) \prime} s$ are the Pauli matrices and the total filling factor is

$$
\widetilde{\nu}=\frac{1}{N_{\varphi}} \sum_{\xi, \alpha, n, X}\left\langle c_{\xi, \alpha, n, X}^{\dagger} c_{\xi, \alpha, n, X}\right\rangle .
$$

Note that these 10 fields do not provide a complete description of a state. One must also consider the 54 other combinations of indices (the 64 order parameters are not all independent, however). We will use both $S_{i}, L_{i}, O_{i}$ and the order parameters $\left\langle\rho_{n, m}^{a, b}\right\rangle$ to characterize the ground states of the C2DEG.

\section{E. Induced dipoles}

The coupling of the C2DEG with a uniform external electric field in the plane of the layers is given by

$$
H_{E}=-e \int d \mathbf{r} n(\mathbf{r}) \phi(\mathbf{r})
$$

where $\mathbf{E}_{\text {ext }}=-\nabla \phi(\mathbf{r})$. The total density is given by

$$
n(\mathbf{r})=\sum_{\sigma, \xi, n, m} \Psi_{\xi, \sigma, n}^{\dagger}(\mathbf{r}) \Psi_{\xi, \sigma, m}(\mathbf{r}) .
$$

Fourier transforming Eq. (67) and using the form factors defined in Eqs. (44 47), we can show that in an homogeneous state ${ }^{20,25}, H_{E}$ gives the dipolar coupling

$$
H_{E}=-\mathbf{d} \cdot \mathbf{E}_{e x t},
$$

with the total dipole vector defined by $\mathbf{d}=\sum_{a} \mathbf{d}_{a}$ where

$$
\mathbf{d}_{a}=-\sqrt{2} \ell e N_{\varphi}\left(\rho_{x}^{a, a}(0) \widehat{\mathbf{x}}-\rho_{y}^{a, a}(0) \widehat{\mathbf{y}}\right)
$$

is the dipole moment in valley $a_{\xi}$ with spin $a_{\sigma}$. We have defined here

$$
\begin{aligned}
& \rho_{x}^{a}=\frac{1}{2}\left(\rho_{0,1}^{a, a}+\rho_{1,0}^{a, a}\right), \\
& \rho_{y}^{a}=\frac{1}{2 i}\left(\rho_{0,1}^{a, a}-\rho_{1,0}^{a, a}\right) .
\end{aligned}
$$

It is possible to control the orientation of the orbital pseudospins in the $x-y$ plane with an external electric field.

\section{F. Electromagnetic absorption}

The total current operator in second quantization is given by $\mathbf{J}=\sum_{a} \mathbf{J}_{a}$ with

$$
\mathbf{J}_{a}=\sum_{n, m} \int d \mathbf{r} \Psi_{a, n}^{\dagger}(\mathbf{r}) \mathbf{j}_{a}(\mathbf{r}) \Psi_{a, m}(\mathbf{r}),
$$

The current operator $\mathbf{j}_{a}(\mathbf{r})$ is derived from the Hamiltonian in Eq. (9) by making the Peierls substitution $\mathbf{p} \rightarrow \mathbf{P}=\mathbf{p}+e \mathbf{A}_{\text {ext }} / c$ and then taking the derivative with respect to the external vector potential $\mathbf{A}_{\text {ext }}$

$$
\mathbf{j}_{a, i}=-\left.c \frac{\partial H_{a}^{0}}{\partial A_{i}^{e}}\right|_{A_{i}^{e} \rightarrow 0}
$$

where $i=x, y$. This gives

$$
\mathbf{J}=\frac{1}{\hbar} \sum_{a} \Delta_{a}^{0}\left(\widehat{\mathbf{z}} \times \mathbf{d}_{a}\right)=\frac{d \mathbf{d}}{d t},
$$

with

$$
\Delta_{a}^{0}=E_{a, 1}^{0}-E_{a, 0}^{0}=\zeta_{1}+a_{\xi} \beta \Delta_{B}
$$

i.e. the bare gap in valley $\xi$ and

$$
\frac{d \mathbf{d}}{d t}=-\frac{i}{\hbar}\left[H_{H F}^{0}, \mathbf{d}\right]
$$

where $H_{H F}^{0}=N_{\varphi} E_{a, n}^{0} \rho_{n, n}^{a, a}$ is the noninteracting Hamiltonian.

To compute the electromagnetic absorption per unit area, we define the two-particle current-current Green's function

$$
\chi_{J_{\alpha}, J_{\beta}}(\tau)=-\frac{1}{S}\left\langle T_{\tau} J_{\alpha}(\tau) J_{\beta}(0)\right\rangle,
$$

which gives

$$
\begin{aligned}
\chi_{J_{\alpha}, J_{\beta}}\left(i \Omega_{n}\right)= & \left(\frac{e \ell}{\hbar}\right)^{2} \frac{1}{\pi \ell^{2}} \sum_{a, b} \Delta_{a}^{0} \Delta_{b}^{0} \\
& \times \chi_{\rho \bar{\alpha}, \rho \bar{\beta}}^{a, a, b, b}\left(\mathbf{q}=0, i \Omega_{n}\right),
\end{aligned}
$$

where $\alpha, \beta=x, y$ and $\bar{x}=y, \bar{y}=x$. Using Eqs. (71/72), we have for example

$$
\chi_{\rho_{x}, \rho_{x}}^{a, b, c, d}=\frac{1}{4}\left(\chi_{1,0,1,0}^{a, b, c, d}+\chi_{0,1,1,0}^{a, b, c, d}+\chi_{1,0,0,1}^{a, b, c, d}+\chi_{0,1,0,1}^{a, b, c, d}\right)
$$

and similarly for the other components. The absorption can only involve these four combinations of orbital indices whatever the polarization of the electric field of the electromagnetic wave. The retarded current-current response function $\chi_{J_{\alpha}, J_{\beta}}(\omega)$ is obtained from the analytic continuation $i \Omega_{n} \rightarrow \omega+i \delta$ and the electromagnetic absorption for an electromagnetic wave of amplitude $E_{0}$ linearly polarized in the direction $\alpha$ is given by

$$
P_{\alpha}(\omega)=-\frac{1}{\hbar} \Im\left[\frac{\chi J_{\alpha}, J_{\alpha}(\omega)}{\omega}\right] E_{0}^{2} .
$$


This formula is valid at finite frequency only since we have neglected the diamagnetic contribution to the current.

\section{G. Absorption in the incoherent phases}

If there is no coherence in a phase, then

$$
\left\langle\rho_{n, m}^{a, b}\right\rangle=\left\langle\rho_{n, n}^{a, a}\right\rangle \delta_{n, m} \delta_{a, b} .
$$

In this case, we can solve analytically for the absorption because this restriction leads, from Eq. (62) to

$$
\begin{aligned}
& \chi_{n_{1}, n_{2}, n_{3}, n_{4}}^{(0) a, b, c, d}\left(\mathbf{q}, \Omega_{n}\right) \\
= & \chi_{n_{1}, n_{2}, n_{2}, n_{1}}^{(0) a, b, a}\left(\mathbf{q}, \Omega_{n}\right) \delta_{a, d} \delta_{b, c} \delta_{n_{1}, n_{4}} \delta_{n_{2}, n_{3}} .
\end{aligned}
$$

Now, at $\mathbf{q}=0$ the only nonzero Fock interactions in the GRPA equations are given in Eqs. (49) w15 while the only Hartree interactions that need to be considered are those of the form

$$
H_{0,0,0,0}^{\xi,-\xi}(0), H_{1,1,1,1}^{\xi,-\xi}(0), H_{0,0,1,1}^{\xi,-\xi}(0), H_{1,1,0,0}^{\xi,-\xi}(0) .
$$

These interlayer Hartree interaction involves combinations of the form $e^{-q d} / q$ that give a finite contribution at $\mathbf{q}=0$ (and also a diverging contribution that is cancelled by the other terms). It follows that Eq. (60) gives for the GRPA response functions $\chi_{1,0,1,0}^{a, a, c, c}(\omega)=\chi_{0,1,0,1}^{a, a, c, c}(\omega)=0$ and

$$
\chi_{0,1,1,0}^{a, a, c, c}(\omega)=\frac{\chi_{0,1,1,0}^{(0) a, a, a, a}(\omega) \delta_{a, c}}{\left[1+\frac{1}{\hbar} X_{1,1,0,0}^{\left(a_{\xi}, \xi_{\xi}\right)}(0) \chi_{0,1,1,0}^{(0) a, a, a, a}(\omega)\right]},
$$

i.e. valley and spin must be conserved in an optically active electronic transition. Since

$$
\chi_{0,1,1,0}^{(0) a, a, a, a}(\omega)=\frac{\left\langle\rho_{0,0}^{a, a}\right\rangle-\left\langle\rho_{1,1}^{a, a}\right\rangle}{\omega+i \delta-\left(\Delta_{a}^{0}+U_{0,0}^{a, a}-U_{1,1}^{a, a}\right) / \hbar}
$$

(and a similar expression with $0 \rightleftharpoons 1$ for $\chi_{1,0,0,1}^{(0) a, a, a}(\omega)$ ), we have easily

$$
\chi_{0,1,1,0}^{a, a, a, a}(\omega)=\frac{\left\langle\rho_{0,0}^{a, a}\right\rangle-\left\langle\rho_{1,1}^{a, a}\right\rangle}{\omega+i \delta-\left[\Delta_{a}^{0}+\frac{\Delta_{C}}{4}\left\langle\rho_{1,1}^{a, a}\right\rangle\right] / \hbar}
$$

and

$$
\chi_{1,0,0,1}^{a, a, a, a}(\omega)=\frac{\left\langle\rho_{1,1}^{a, a}\right\rangle-\left\langle\rho_{0,0}^{a, a}\right\rangle}{\omega+i \delta+\left[\Delta_{a}^{0}+\frac{\Delta_{C}}{4}\left\langle\rho_{1,1}^{a, a}\right\rangle\right] / \hbar} .
$$

The functions $\chi_{0,1,1,0}^{a, a, a, a}(\omega)$ and $\chi_{1,0,0,1}^{a, a, a, a}(\omega)$ are the response to the two circular polarizations of light. The absorption in an incoherent phase is finally given by

$$
\begin{aligned}
P_{\alpha}(\omega)= & \frac{E_{0}^{2}}{4 \ell^{2} \omega}\left(\frac{e \ell}{\hbar}\right)^{2} \sum_{a}\left(\left\langle\rho_{0,0}^{a, a}\right\rangle-\left\langle\rho_{1,1}^{a, a}\right\rangle\right) \\
& \times\left(\Delta_{a}^{0}\right)^{2} \delta\left(\hbar \omega-\Delta_{a}^{0}-\frac{\Delta_{C}}{4}\left\langle\rho_{1,1}^{a, a}\right\rangle\right)
\end{aligned}
$$

with $\alpha=x, y$ and $\Delta_{a}^{0}$ given by Eq. (76). In the numerical calculation, we introduce a small Landau level width in order to get a finite value for the optical absorption.

We can follow the same type of reasoning to show that, in a phase with no orbital coherence but with possibly layer and/or spin coherence, the functions $\chi_{1,0,1,0}^{a, b, c, d}(\omega)=$ $\chi_{0,1,0,1}^{a, b, c, d}(\omega)=0$ and the absorption depends again only on $\chi_{0,1,1,0}^{a, a, b}(\omega)$ and $\chi_{1,0,0,1}^{a, a, b, b}(\omega)$. In this special case, the equation of motion for $\chi_{0,1,1,0}^{a, b, c, d}(\omega)$ is

$$
\begin{aligned}
\chi_{0,1,1,0}^{a, a, b, b}(\omega)= & \chi_{0,1,1,0}^{(0) a, a, b, b}(\omega) \\
& -\frac{1}{\hbar} \sum_{e, f} \chi_{0,1,1,0}^{(0) a, a, e, f}(\omega) X_{1,1,0,0}^{e_{\xi}, f_{\xi}} \chi_{0,1,1,0}^{f, e, b, b}(\omega)
\end{aligned}
$$

and a similar expression with $0 \rightleftharpoons 1$ for $\chi_{1,0,0,1}^{a, b, c, d}(\omega)$.

\section{PHASE DIAGRAM OF THE C2DEG}

At zero bias, the QHF states follow a set of Hund's rules: the spin polarization is maximized first, then the layer polarization is maximized to the greatest extent possible and finally the orbital polarization is maximized to the extent allowed by the first two rules 13 . In this section, we study the phase transitions that occur when a finite bias (or transverse electric field) is turned on.

\section{A. Types of phases}

Fig. [5 shows our numerical result for the phase diagram of the C2DEG as a function of an applied transverse electric field $E=\Delta_{B} / e d$ for $B=10 \mathrm{~T}$ and $\kappa=5$ and for all integer filling factors $\nu \in[-3,3]$. We indicate the eight non-interacting levels by horizontal lines and number them according to the scheme indicated in the top inset. Note that the lines are only offset vertically for clarity. Their position does not reflect the true ordering of the energy levels which changes with bias. We name the phases $I_{\nu}, L_{\nu}, O_{\nu}$ and $S L_{\nu}$ according to the type of coherence that is present: incoherent, layer-coherent, orbitalcoherent or spin-layer coherent respectively. When there is more than one incoherent phase at a given filling factor, we use the notation $I_{\nu}^{*}$ for the second phase, $I_{\nu}^{* *}$ for the third phase and so on. The critical electric field for the transition between two phases is indicated by $E^{(1)}$ to $E^{(18)}$ and is in units of $\mathrm{mV} / \mathrm{nm}$. A circle on an energy level represents a fully filled level while an ellipse that connects two levels indicates a coherent superposition of these two states. We list in Fig. [5]some properties of each phase: spin polarization $S_{z}$, number of Goldstone modes (G), number of collective modes gapped at the Zeeman energy (Z) and number of peaks in the optical absorption spectrum (A). The transition between a coherent and an incoherent phase is continuous while a transition between two incoherent phases is discontinuous. 


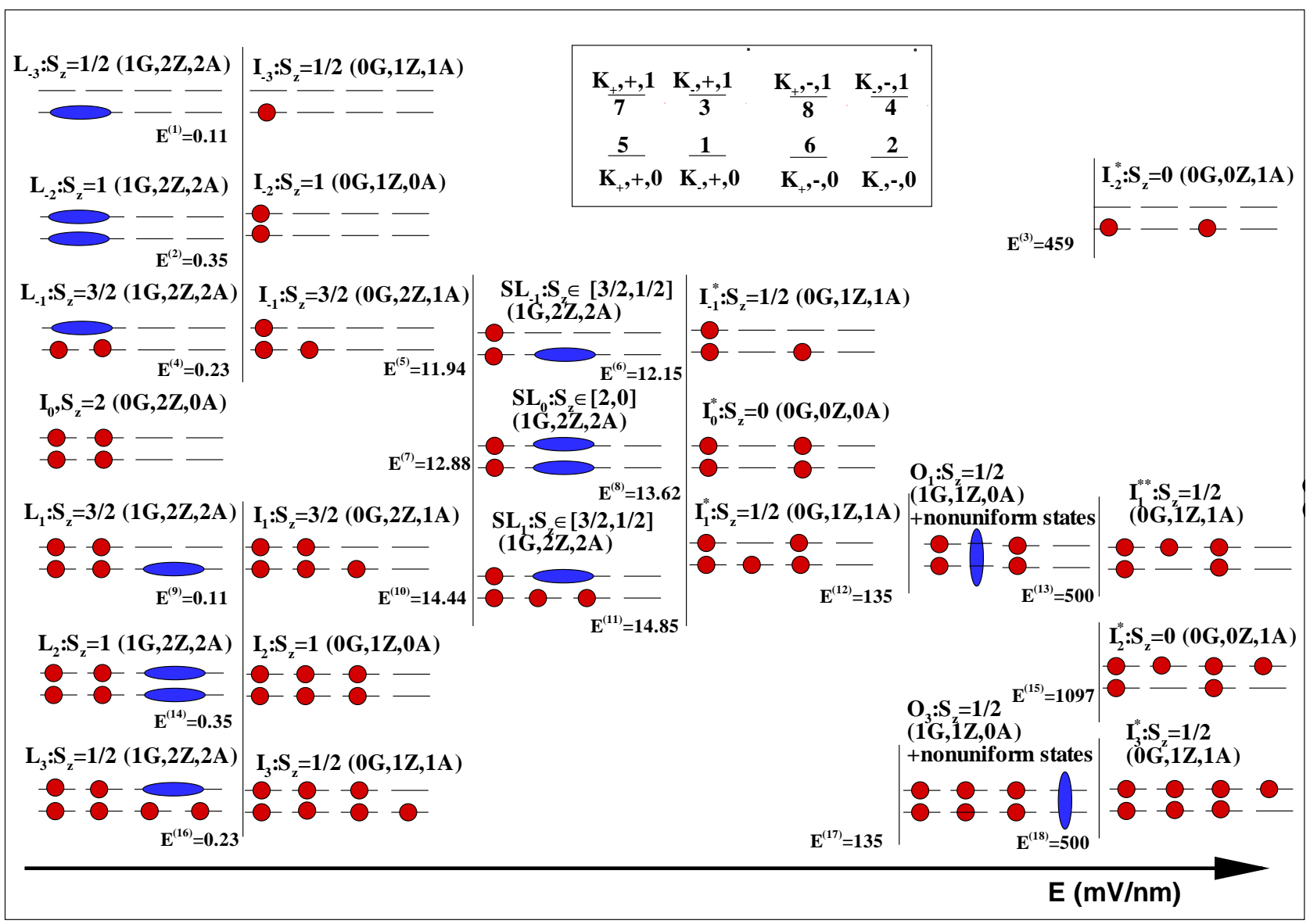

FIG. 5: (Color online) Phase diagram of the C2DEG in Landau level $N=0$ at $B=10 \mathrm{~T}$ and for $\kappa=5$ as a function of the transverse electric field beween the layers for integer filling factors $\nu \in[-3,3]$ (from top to bottom). A filled (red) circle represents a filled state while a filled (blue) ellipse indicates a coherent superposition of two levels. The numbering of the levels is indicated in the inset at the top right of the figure. The $E^{(i) \prime} s$ indicate the critical perpendicular electric field (in $\mathrm{mV} / \mathrm{nm}$ ) required for the transition between two phases. Also indicated for each phase are the spin polarization $S_{z}$, the number of Goldstone mode $(\mathrm{G})$, of modes gapped at the Zeeman energy $(\mathrm{Z})$ and the number of peaks in the optical absorption spectrum (A). The mention "+ nonuniform states" for the $O_{1}$ and $O_{3}$ phases signals that this portion of the phase diagram is further subdivided into uniform and nonuniform states as indicated in Eq. (109).

\section{Incoherent phases $I_{\nu}$}

The $I_{\nu}$ phases have no coherence of any kind and so $L_{\|}, S_{\|}, O_{\|}=0$ (the parallel component is in the plane of the bilayer). Each level is either full or empty and so $L_{z}, S_{z}$ and $O_{z}$ vary from one phase to another. The state corresponding to a specific diagram is easily read from Fig. 5. For $I_{2}$, we have for example

$$
\left|\Psi_{I_{2}}\right\rangle=\prod_{X} c_{7, X}^{\dagger} c_{5, X}^{\dagger}|0\rangle
$$

and the order parameters $\left\langle\rho_{5,5}\right\rangle=\left\langle\rho_{7,7}\right\rangle=1$. We include the phases $I_{ \pm 2}^{*}, I_{1}^{* *}$ and $I_{3}^{*}$ in the phase diagram only to make it more complete. Indeed, the bias can't produce any more transition after these states. But, the critical bias needed to reach these states is well outside the limits of validity of our two-band model.

Using Eq. (48), the Hartree-Fock energy of two adja- cent incoherent phases are readily compared to extract the critical biases. We find, with $\Delta_{B}^{(i)}=e d E^{(i)}$,

$$
\begin{aligned}
& I_{-2} \rightarrow I_{-2}^{*}: \Delta_{B}^{(3)}=\frac{\Delta_{Z}-\zeta_{1}+\frac{3}{8} \Delta_{C}}{\beta} \\
& I_{+2} \rightarrow I_{+2}^{*}: \Delta_{B}^{(15)}=\frac{\Delta_{Z}+\zeta_{1}+\frac{5}{8} \Delta_{C}}{\beta} .
\end{aligned}
$$

If we ignore the $S L_{\nu}$ phases at $\nu=0, \pm 1$, we find that the transition between the two incoherent phases occurs in the middle of the $S L_{\nu}$ phase (see Sec. IV.A.4 below) 
i.e. at

$$
\begin{gathered}
I_{-1} \rightarrow I_{-1}^{*}: \Delta_{-1}=\Delta_{z}+2 \frac{d}{\ell} \alpha, \\
I_{0} \rightarrow I_{0}^{*}: \Delta_{0}=\frac{\Delta_{z}+2 \frac{d}{\ell} \alpha}{1-\beta}, \\
I_{1} \rightarrow I_{1}^{*}: \Delta_{1}=\frac{\Delta_{z}+2 \frac{d}{\ell} \alpha}{1-2 \beta} .
\end{gathered}
$$

Note that these last three results are independent of Coulomb exchange corrections and so of screening corrections. (The capacitive term comes from the Hartree self-energy and is not screened.)

From Eq. (95), we find a critical electric field $E_{c}=$ $\Delta_{0} / e d \approx 4.7 B[\mathrm{~T}] \mathrm{mV} / \mathrm{nm}$ when $\kappa=1$ for the transition $I_{0} \rightarrow I_{0}^{*}$. This critical field depends linearly on the magnetic field, in agreement with the experiments 12 . Experimentally, however, the slope is $1211 \mathrm{mV} \mathrm{nm}^{-1} \mathrm{~T}^{-1}$ or $^{10} 12.7 \mathrm{mV} \mathrm{nm}^{-1} \mathrm{~T}^{-1}$ or $^{14} 12-18 \mathrm{mV} \mathrm{nm}^{-1} \mathrm{~T}^{-1}$ and thus larger than the HFA value. Moreover, experiments measure an offset of $E_{c} \approx 20 \mathrm{mV} / \mathrm{nm}$ at $B=0 \mathrm{~T}^{12}$. This offset can't be captured by our HFA which is only valid at sufficiently large magnetic field where Landau level mixing can be neglected. Apart from the extra $\beta$ corrections, Eqs. (94, $\sqrt{96}$ are identical to those given by Gorbar et al. 17 .

\section{Layer-coherent phases $L_{\nu}$}

The second type of phase, $L_{\nu}$, has layer coherence between two states with the same spin and orbital indices and so $L_{\|} \neq 0$ but $S_{\|}, O_{\|}=0$. The tilt angle of the pseudospin vector $\mathbf{L}$ varies with bias in this phase but $O_{z}$ and $S_{z}$ are constant. An example is phase $L_{-3}$ which is described by

$$
\left|\Psi_{L_{-3}}\right\rangle=\prod_{X}\left(a c_{5, X}^{\dagger}+b c_{1, X}^{\dagger}\right)|0\rangle,
$$

where the coefficients $a$ and $b$ depend on the bias and are related by $|a|^{2}+|b|^{2}=1$. With increasing bias, $a \rightarrow 1$ and $b \rightarrow 0$ continuously. The level populations and the coherence in $L_{-3}$ are given by

$$
\begin{aligned}
& \left\langle\rho_{5,5}\right\rangle=|a|^{2},\left\langle\rho_{1,1}\right\rangle=|b|^{2} \\
& \left\langle\rho_{1,5}\right\rangle=\left\langle\rho_{5,1}\right\rangle^{*}=a b^{*} .
\end{aligned}
$$

Fig. 6] shows how these variables depend on the transverse electric field for the similar phase $L_{-1}$. The populations of the coherent levels vary linearly with the bias in all $L_{\nu}$ phases with the exception of $L_{ \pm 2}$ where the variation is not exactly linear. In $L_{-1}$, for example,

$$
\left\langle\rho_{5,5}\right\rangle=\frac{1}{2}\left(1+\frac{\Delta_{B}}{\Delta_{B}^{(1)}}\right),
$$

where the critical bias is, to order $(d / \ell)^{2}$,

$$
\begin{aligned}
L_{-3(1)} & \rightarrow I_{-3(1)}: \Delta_{B}^{(1)}=\Delta_{B}^{(9)} \approx \sqrt{\frac{\pi}{8}}\left(\frac{d}{\ell}\right)^{2} \alpha \\
L_{-1(3)} & \rightarrow I_{-1(3)}: \Delta^{(4)}=\Delta_{B}^{(16)} \\
& \approx \frac{7}{4} \sqrt{\frac{\pi}{8}}\left(\frac{d}{\ell}\right)^{2} \alpha .
\end{aligned}
$$

These critical biases all scale with the magnetic field as $B^{3 / 2}$.

In phases $L_{ \pm 2}$, there is a layer coherence in orbitals $n=0$ and $n=1$. Phase $L_{-2}$, for example, is described by the state

$$
\begin{aligned}
\left|\Psi_{L_{-2}}\right\rangle= & \prod_{X}\left(\alpha c_{5, X}^{\dagger}+\gamma c_{1, X}^{\dagger}\right) \\
& \times\left(\alpha^{\prime} c_{7, X}^{\dagger}+\gamma^{\prime} c_{3, X}^{\dagger}\right)|0\rangle .
\end{aligned}
$$

The critical bias $\Delta^{(2)}=\Delta^{(14)}$ for the transitions $L_{ \pm 2} \rightarrow$ $I_{ \pm 2}$ has a complicated analytical expression that we do not reproduce here but the numerical values of the critical electric field for $B=10 \mathrm{~T}$ and $\kappa=5$ is indicated in Fig. 5.

Phases with layer coherence occur in a small range of bias and at very small bias because the interlayer separation $d / \ell=0.013 \sqrt{B}$ is very small in bilayer graphene and so is the capacitive energy. In semiconductor bilayers, $d / \ell$ can be of order unity and interlayer coherence can survive to a much higher bias 31 .

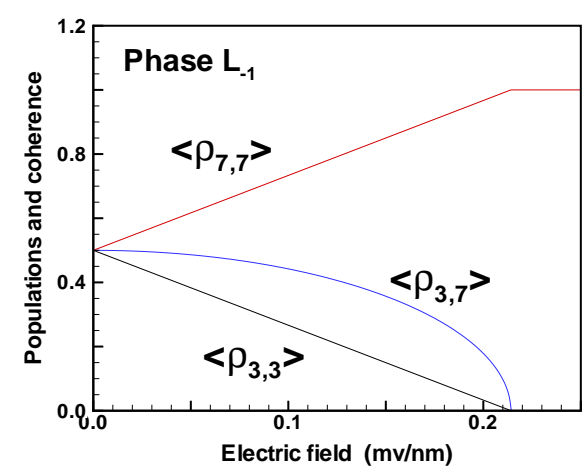

FIG. 6: (Color online) Variation of the populations and interlayer coherence with the transverse electric field in phase $L_{-1}$.

\section{Orbital-coherent phases $O_{\nu}$}

For $\Delta_{B}>\Delta_{B}^{(12)}=\Delta_{B}^{(17)}$, the ordering of the energy levels $n=0,1$ is reversed (this change is not shown in Fig. 5). When this happens, the kinetic energy is minimized by filling level $n=1$ before $n=0$. However, this increases the Coulomb exchange energy because 
$X_{1,1,1,1}^{+,+}(0)<X_{0,0,0,0}^{+,+}(0)$. The C2DEG optimizes its energy by creating a coherent superposition of $n=0$ and $n=1$ with the same valley and spin indices. We use the notation $O_{\nu}$ for such a phase. An example is phase $\mathrm{O}_{3}$ which is described by

$$
\begin{aligned}
\left|\Psi_{O_{3}}\right\rangle= & \prod_{X}\left(a c_{2, X}^{\dagger}+b c_{4, X}^{\dagger}\right) c_{8, X}^{\dagger} c_{6, X}^{\dagger} \\
& \times c_{7, X}^{\dagger} c_{5, X}^{\dagger} c_{3, X}^{\dagger} c_{1, X}^{\dagger}|0\rangle .
\end{aligned}
$$

In this state, $L_{\|}=S_{\|}=0$ and $L_{z}$ and $S_{z}$ are constant. It is now the tilt angle of the pseudospin vector $\mathbf{O}$ that varies with bias. Orbital coherence begins when the bare energy of the state $\left|K_{-}, \pm, 0\right\rangle$ is equal to that of state $\left|K_{-}, \pm, 1\right\rangle$ at $\nu=1,3$. This occurs when

$$
\begin{aligned}
& I_{1}^{*} \rightarrow O_{1}: \Delta_{B}^{(12)}=\frac{\zeta_{1}}{\beta} \\
& I_{3} \rightarrow O_{3}: \Delta_{B}^{(17)}=\Delta_{B}^{(12)}
\end{aligned}
$$

and the critical bias does not depend on the magnetic field, Coulomb interaction or on the value of the dielectric constant. We find $\zeta_{1} / \beta=46 \mathrm{meV}$ i.e. $E=135 \mathrm{mV} / \mathrm{nm}$ which is in the range of validity of the two-band model.

The orbital phase survives until a transition to an incoherent phase occurs at the critical bias

$$
\begin{aligned}
& O_{1} \rightarrow I_{1}^{* *}: \Delta_{B}^{(13)}=\frac{1}{\beta}\left(\zeta_{1}+\frac{1}{4} \Delta_{C}\right), \\
& O_{3} \rightarrow I_{3}^{*}: \Delta_{B}^{(18)}=\Delta_{B}^{(13)}
\end{aligned}
$$

Since $\zeta_{1} / \beta$ is the onset of the orbital phase, we see that the range of existence of the orbital phase scales as $1 / \sqrt{B}$. Part of this range is outside the limit of validity of our model.

Fig. 7 shows how the populations and coherence vary with the transverse electric field in phase $O_{3}$. The same behavior is found in phase $O_{1}$. We remark that in previous work where the spin degree of freedom is frozen ${ }^{25}$, the orbital coherent phase occurs at $\nu=-1$ and $\nu=3$.

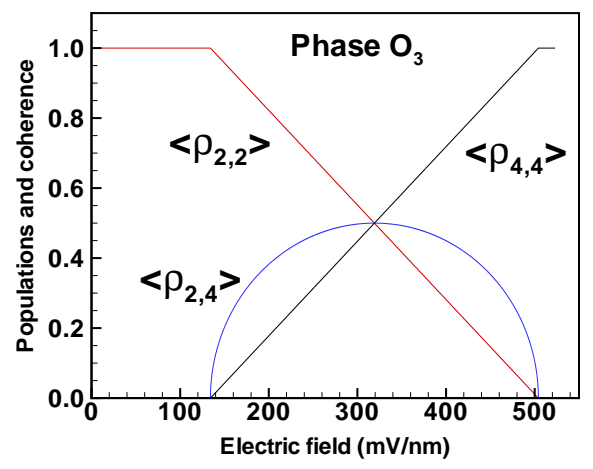

FIG. 7: (Color online) Variation of the populations interlayer coherence with the transverse electric field in phase $O_{3}$.
The orbital phase exists in a large range of bias. As we explained in Sec. III.E, a finite orbital coherence implies a finite density of electric dipoles in the plane of the layers. The orientation of these dipoles can be controlled by an electric field in the plane of the layers ${ }^{20}$. On of us has studied in detail the interesting properties of this state 25,26 . For example, the collective mode associated with the orbital coherence is highly anisotropic. This mode softens at a finite wave vector in the direction perpendicular to the dipoles when the bias is increased. This suggests a transition to a charge-density-wave state. In the Hartree-Fock approximation, it was found that this transition is preempted by a transition to a crystal phase with one electron per site and a Skyrmion-like pseudospin texture of the orbital pseudospin at each crystal site. As the bias is increased, the crystal state is followed by a helical state where the orbital pseudospin rotates along one spatial direction. In both phases, the total electronic density is modulated spatially but the local filling factor is not. By further increasing the bias, the crystal state and then the uniform states are recovered. The critical electric fields for the transition to the uniform (UP), Skyrmion crystal (SKP) and helical phases (HP) are given by 26,32 :

\begin{tabular}{|l|l|}
\hline $134<\Delta_{B}<145 \mathrm{mV} / \mathrm{nm}$ & $\mathrm{UP}$ \\
\hline $145<\Delta_{B}<189 \mathrm{mV} / \mathrm{nm}$ & $\mathrm{SKP}$ \\
\hline $189<\Delta_{B}<450 \mathrm{mV} / \mathrm{nm}$ & $\mathrm{HP}$ \\
\hline $450<\Delta_{B}<494 \mathrm{mV} / \mathrm{nm}$ & $\mathrm{SKP}$ \\
\hline $494<\Delta_{B}<505 \mathrm{mV} / \mathrm{nm}$ & $\mathrm{UP}$ \\
\hline
\end{tabular}

The phase diagram in $O_{1}$ and $O_{3}$ is symmetric with respect to the center of the helical phase.

We remark that this sequence of phase transitions is similar to that observed in a thin film of the helical magnet $\mathrm{Fe}_{0.5} \mathrm{Co}_{0.5} \mathrm{Si}$ when a perpendicular magnetic field is increased ${ }^{33}$. It has been shown ${ }^{26}$ that the Hamiltonian of the C2DEG in the orbital phase contains a Dzyaloshinskii-Moriya (DM) interaction ${ }^{34}$ that is responsible for the rotation of the pseudospins. Its origin in bilayer graphene is purely Coulombic while the DM interaction comes from spin-orbit coupling in $\mathrm{Fe}_{0.5} \mathrm{Co}_{0.5} \mathrm{Si}$.

We do not find any sign of instability in the collective mode dispersions for the other phases in Fig. 5. However, we remark that phases with lower energy than those considered in this figure are possible. In order to establish the phase diagram of the C2DEG, we choose a set of possible ground states and compare their energies. This does not ensure however that the true ground state is amongst the states that we have chosen to compare! For the incoherent states, this is not a problem because there is a finite number of states to compare. But for the nonuniform states, the number of possible ground state is enormous. 


\section{Spin-layer-coherent phases $S L_{\nu}$}

The fourth type of phase has coherence between two states with the same orbital index but different spin and layer indices. We use for these phases the notation $S L_{\nu}$. An example is phase $S L_{-1}$ where the ground state is

$$
\left|\Psi_{S L_{-1}}\right\rangle=\prod_{X}\left(a c_{6, X}^{\dagger}+b c_{1, X}^{\dagger}\right) c_{7, X}^{\dagger} c_{5, X}^{\dagger}|0\rangle .
$$

Because the coherence is now between two states with different spin and layer indices, i.e. $\left|K_{+},-, 0\right\rangle$ and $\left|K_{-},+, 0\right\rangle$ in $\left|\Psi_{S L_{-1}}\right\rangle$, we cannot describe the change with bias as the tilting of one of the pseudospin $\mathbf{L}, \mathbf{S}$ or O. In fact this state has $L_{\|}, S_{\|}=O_{\|}=0$. Both $L_{z}$ and $S_{z}$ vary with bias however. This phase is characterized by the order parameter $\left\langle\rho_{6,1}\right\rangle$.

The variation of $\left\langle\rho_{6,6}\right\rangle,\left\langle\rho_{1,1}\right\rangle$ and $\left\langle\rho_{6,1}\right\rangle$ with bias in this phase is similar to that shown in Fig. 7]. The critical biases for the beginning $\left(\Delta_{b}(n)\right)$ and end $\left(\Delta_{e}(n)\right)$ of the $S L_{ \pm 1}$ phases are given by

$$
\begin{aligned}
& \Delta_{b}(n)=\frac{\frac{d}{\ell} \alpha+\Delta_{z}+X_{n}}{1-2 \beta \delta_{n, 1}}, \\
& \Delta_{e}(n)=\frac{3 \frac{d}{\ell} \alpha+\Delta_{z}-X_{n}}{1-2 \beta \delta_{n, 1}},
\end{aligned}
$$

where $X_{n}=X_{n, n, n, n}^{+,+}(0)-X_{n, n, n, n}^{+,-}(0)$. Thus,

$$
\begin{aligned}
& \Delta^{(5)} \approx\left(2 \frac{d}{\ell}-\sqrt{\frac{\pi}{8}}\left(\frac{d}{\ell}\right)^{2}\right) \alpha+\Delta_{z}, \\
& \Delta^{(6)} \approx\left(2 \frac{d}{\ell}+\sqrt{\frac{\pi}{8}}\left(\frac{d}{\ell}\right)^{2}\right) \alpha+\Delta_{z}, \\
& \Delta^{(10)} \approx \frac{\left(2 \frac{d}{\ell}-7 \sqrt{\frac{\pi}{128}}\left(\frac{d}{\ell}\right)^{2}\right) \alpha+\Delta_{z}}{1-2 \beta}, \\
& \Delta^{(11)} \approx \frac{\left(2 \frac{d}{\ell}+7 \sqrt{\frac{\pi}{128}}\left(\frac{d}{\ell}\right)^{2}\right) \alpha+\Delta_{z}}{1-2 \beta} .
\end{aligned}
$$

These critical biases scale linearly with the magnetic field. The phase $S L_{\nu}$ is the ground state in a small range of bias of the order $(d / \ell)^{2} \alpha$ which is approximately 0.1 meV for $\kappa=5$.

A sufficiently large bias is necessary for spin down states to cross the spin up states and produces a $S L_{\nu}$ phase. The $S L_{0}$ is special because it involves coherence in both $n=0$ and $n=1$. As for $L_{ \pm 2}$, the exact critical bias in this case has a complicated analytical expression which we do not reproduce here.

\section{B. Spin polarization}

We indicate for each phase in Fig. 5 the spin polarization $S_{z}$. The polarization is constant in all phases with the exception of the phases $S L_{\nu}$ where it varies continuously between the two numbers indicated. The biggest change in $S_{z}$ and $L_{z}$ occurs at filling factor $\nu=0$ where the C2DEG makes a transition from a fully spin polarized and layer unpolarized gas $\left(S_{z}=2 \hbar, L_{z}=0\right)$ at small bias to a spin unpolarized and layer polarized gas $\left(S_{z}=0, L_{z}=2\right)$ at large bias. For $\nu= \pm 1$, the $S L_{\nu}$ phase interpolates between $S_{z}=3 \hbar / 2, L_{z}=1 / 2$ and $S_{z}=\hbar / 2, L_{z}=3 / 2$. The only jumps in $S_{z}$ occur at the transitions $I_{ \pm 2} \rightarrow I_{ \pm 2}^{*}$ where the system goes from a spin polarized to a spin unpolarized state.

\section{Transport gaps}

Another quantity that is accessible experimentally is the transport gap $\Delta$ which is defined by the difference in energy between the first empty state and the last filled state of the Hartree-Fock Hamiltonian. It was shown previously 13 that the gap at zero bias follow the hierarchy $\Delta_{\nu=0}>\Delta_{\nu= \pm 2}>\Delta_{\nu= \pm 1, \pm 3}$. This implies that the first plateau to appear when the magnetic field is turned on has $\sigma_{x y}=0$. At larger field, the $\sigma_{x y}= \pm 2$ plateaus appear and at still larger field, the $\sigma_{x y}= \pm 1, \pm 3$ plateaus. This is indeed what is seen experimentally $\underline{5,6,9}$.

Fig. 8 shows the Hartree-Fock gaps as a function of the transverse electric field for the different phases of the C2DEG. For this figure, we have taken $B=10 \mathrm{~T}$ and $\kappa=$ 5 . In some phases, one or more level crossing occurs that change the behavior of the gap. This is clearly visible for $I_{ \pm 2}$ in Fig. 8 (a) and for $I_{-1}$ in Fig. 8 (c).

With the exceptions of the phases where coherence occurs in two levels $\left(L_{ \pm 2}, S L_{0}\right)$, it is possible to obtain a simple analytical expression for the gap. We list these expressions below. When one or more level crossings occur, we use the notation $I_{\nu}^{(1)}, I_{\nu}^{(2)}, I_{\nu}^{(3)}, \ldots$ to denote the different behaviors of the gap and $\Delta_{B}^{(j)-(j+1)}$ for the values at which the level crossings occur.

In the incoherent phases with $\nu= \pm 1, \pm 3$ :

$$
\begin{gathered}
I_{-3}, I_{1}, I_{-1}^{*(1)}: \Delta=\beta \Delta_{B}+\zeta_{1}+\frac{1}{2} \Delta_{C}, \\
I_{3}^{*}, I_{1}^{* *}: \Delta=\beta \Delta_{B}-\zeta_{1}+\frac{1}{4} \Delta_{C} . \\
I_{-1}, I_{1}^{*}, I_{3}: \Delta=-\beta \Delta_{B}+\zeta_{1}+\frac{1}{2} \Delta_{C}, \\
I_{-1}^{*(2)}: \Delta_{-1}^{*}=\Delta_{Z}+\frac{3}{4} \Delta_{C},
\end{gathered}
$$

where, for $\nu=-1$ :

$$
\Delta_{B}^{(1)-(2)}=\frac{\Delta_{z}-\zeta_{1}+\frac{1}{4} \Delta_{C}}{\beta} .
$$


For the incoherent phases with $\nu=-2$

$$
\begin{aligned}
& I_{-2}^{(1)}: \Delta=(1-\beta) \Delta_{B}-\zeta_{1}-2 \frac{d}{\ell} \alpha+\frac{5}{4} \Delta_{C} \\
& I_{-2}^{(2)}: \Delta=-\beta \Delta_{B}-\zeta_{1}+\Delta_{Z}-2 \frac{d}{\ell} \alpha+\frac{5}{4} \Delta_{C} \\
& I_{-2}^{*}: \Delta=\beta \Delta_{B}+\zeta_{1}-\Delta_{Z}+\frac{1}{2} \Delta_{C}
\end{aligned}
$$

with

$$
\Delta_{B}^{(1)-(2)}=\Delta_{Z}+2 \frac{d}{\ell} \alpha
$$

while for $\nu=2$,

$$
\begin{aligned}
& I_{2}^{(1)}: \Delta=(1-\beta) \Delta_{B}-\zeta_{1}-2 \frac{d}{\ell} \alpha+\frac{5}{4} \Delta_{C}, \\
& I_{2}^{(2)}: \Delta=\beta \Delta_{B}+\Delta_{Z}-\zeta_{1}+\frac{5}{4} \Delta_{C} \\
& I_{2}^{(3)}: \Delta=\Delta_{Z}+\frac{5}{4} \Delta_{C} \\
& I_{2}^{(4)}: \Delta=-\beta \Delta_{B}+\Delta_{Z}+\zeta_{1}+\frac{3}{2} \Delta_{C}
\end{aligned}
$$

with

$$
\begin{aligned}
\Delta_{B}^{(1)-(2)} & =\frac{2 \frac{d}{\ell} \alpha+\Delta_{z}}{1-2 \beta}, \\
\Delta_{B}^{(2)-(3)} & =\frac{\zeta_{1}}{\beta} \\
\Delta_{B}^{(3)-(4)} & =\frac{\zeta_{1}+\frac{1}{4} \Delta_{C}}{\beta} .
\end{aligned}
$$

For $\nu=0$, we find:

$$
\begin{aligned}
I_{0}: & \Delta=-(1-\beta) \Delta_{B}-\zeta_{1}+\Delta_{Z}+\frac{5}{4} \Delta_{C}, \\
I_{0}^{*(1)}: & \Delta=(1-\beta) \Delta_{B}-\zeta_{1}-\Delta_{Z} \\
& -4 \frac{d}{\ell} \alpha+\frac{5}{4} \Delta_{C} \\
I_{0}^{*(2)}: & \Delta=(1-2 \beta) \Delta_{B}-\Delta_{Z} \\
& -4 \frac{d}{\ell} \alpha+\frac{5}{4} \Delta_{C}
\end{aligned}
$$

with

$$
\Delta_{B}^{(1)-(2)}=\frac{\zeta_{1}}{\beta} .
$$

The gap changes rapidly in the $S L_{ \pm 1}$ phase while it is almost independent of the bias in phase $S L_{0}$. Its value in the middle of the $S L_{ \pm 1}$ phases is given approximately by

$$
\begin{aligned}
S L_{-1}: & \Delta \approx \zeta_{1}+\frac{\Delta_{C}}{2}\left(1-\frac{1}{4}\left(\frac{d}{\ell}\right)^{2}\right) \\
& -4 \beta^{2}\left(\frac{\Delta_{-1}}{\Delta_{C}}\right)^{2} \frac{\Delta_{C}}{2}, \\
S L_{1}: & \approx \zeta_{1}+\frac{\Delta_{C}}{2}\left(1+\frac{1}{8}\left(\frac{d}{\ell}\right)^{2}\right), \\
& -4 \beta^{2}\left(\frac{\Delta_{1}}{\Delta_{C}}\right)^{2} \frac{\Delta_{C}}{2},
\end{aligned}
$$

where $\Delta_{ \pm 1}$ are defined in Eqs. (9496). The gaps are twice as big in phases $I_{0}, I_{ \pm 2}$ than in phases $I_{ \pm 1}, I_{ \pm 3}$ and vary more rapidly with bias in the former than in the latter. The presence of the $S L_{\nu}$ phase smoothens the jump of the gap in the transition from $I_{ \pm 1}$ to $I_{ \pm 1}^{*}$.

The gap is independent of the bias in the orbital phases $\mathrm{O}_{1}$ and $\mathrm{O}_{3}$ :

$$
O_{1}, O_{3}: \Delta=\frac{\Delta_{C}}{2}
$$

For the $L_{\nu}$ phases, the gaps at zero bias are given approximately by

$$
\begin{aligned}
L_{-3}, L_{1}: \Delta & \approx \zeta_{1}+\frac{1}{2}\left(1-\frac{1}{4}\left(\frac{d}{\ell}\right)^{2}\right) \Delta_{C}, \\
L_{-1}, L_{3}: \Delta & \approx \zeta_{1}+\frac{1}{2}\left(1+\frac{1}{8}\left(\frac{d}{\ell}\right)^{2}\right) \Delta_{C}, \\
L_{ \pm 2},: \Delta & \approx-\zeta_{1}-2\left(\frac{d}{\ell}\right) \alpha \\
+ & \frac{1}{4}\left(5+\frac{23}{4}\left(\frac{d}{\ell}\right)^{2}\right) \Delta_{C} .
\end{aligned}
$$

The correction $(d / \ell)^{2}$ is very small and the gaps at zero bias for $L_{ \pm 1}$ and $L_{ \pm 3}$ are almost equal. (The difference comes from the fact that the coherence is not in the same orbital in $L_{-3}, L_{1}$ and $L_{-1}, L_{3}$.) These gaps are not shown in Fig. [5 because the corresponding phases occur at very small biases. The gap increases (decreases) with bias in phases $L_{-3}, L_{1}\left(L_{-1}, L_{3}\right)$. It is almost constant in $L_{ \pm 2}$. The main contribution to all gaps is the Coulomb exchange interaction.

With $\zeta_{1}=0$, our gaps for phases $I_{2}, I_{0}, I_{0}^{*}$ agree with those of Gorbar et al. 17 if screening is neglected in their calculation. For $I_{1}, I_{1}^{*}$ and $I_{3}$, however, our exchange correction is $\Delta_{C} / 2$ which is consistent with Ref. 13 while Gorbar et al. have $3 \Delta_{C} / 8$.

At $\Delta_{B}=0$, we find for $I_{0}$ the gap $\Delta=62 \mathrm{meV}$ for $B=2 \mathrm{~T}$ and $\kappa=2$ while, with static screening, Gorbar et al. find $\approx 5 \mathrm{meV}$. Similarly, for phase $I_{1}$, the HFA gives $\Delta=26 \mathrm{meV}$ while the result with static screening is $\approx 2.5 \mathrm{meV}$. Static screening leads to a reduction of the gap by a factor of at least 10 . Dynamical screening and Landau mixing corrections, however, increase the 
gaps calculated with static screening by a factor of two to three $\frac{18}{}$. As for the behavior of the gap with bias. Fig. 5 of Gorbar et al. ${ }^{17}$ shows that, with screening, the gap of the phase $I_{3}$ increases with the electric field even when the correction $\beta \Delta_{B}$ is neglected. The slope is approximately $0.1 \mathrm{~nm}-\mathrm{C}$ for $B=2 \mathrm{~T}$ and $\kappa=2$. The (unscreened) HFA predicts a slope of $\beta d=0.006 \mathrm{~nm}-\mathrm{C}$. When both screening and $\beta \Delta_{B}$ are considered, the gaps for $I_{ \pm 1}, I_{ \pm 3}, I_{ \pm 1}^{*}$ will probably increase with bias (contrary to the behavior illustrated in our Fig. $8(\mathrm{~b})$ ) but the rapid change of the gap in the $S L_{ \pm 1}$ will still be present.

The energy gaps obtained from local compressibility measurements on suspended bilayer graphene by Martin et al. $\underline{\underline{6}}$ are of size $\Delta_{\nu=0} \approx 1.7 B[\mathrm{~T}] \mathrm{meV}, \Delta_{\nu= \pm 2} \approx 1.2 B$ [T] $\mathrm{meV}$ and $\Delta_{\nu= \pm 1} \approx 0.1 B$ [T] $\mathrm{meV}$ (with less data points in this case). A more recent transport experiment by Velasco et al. ${ }^{10}$ on suspended bilayer graphene with a higher mobility reports a larger gap $\Delta_{\nu=0} \approx 5.5 B[\mathrm{~T}]$ $\mathrm{meV}$. The measured gaps scale linearly with the magnetic field contrary to the HFA prediction. In fact, Gorbar et al. $\frac{17}{1}$ have shown that a linear scaling is obtained if static screening is considered. (One set of experiments at higher magnetic field reported gaps that scaled as $\sqrt{B}$ however ${ }^{9}$.)

Experiments 12 show that $\sigma_{x y}$ ceases to be quantized at $\nu=0,1$ in the region corresponding to the $S L_{\nu}$ phase and at $\nu=2$ and $\nu=3$ in the region around zero bias. A possible explanation is that the conductance quantization is broken by disorder in the regions corresponding to a minimum of the gap 17 . However, that argument does not seem to work at $\nu=1$ where the screened HFA gap increases continuously with the bias and is also not compatible with our unscreened result.

\section{Collective modes and optical absorption}

Each phase of the C2DEG is characterized by a set of collective excitations. The number of dispersive modes when $m$ levels are filled is $m(8-m)$. We have calculated the dispersion relation of these modes using the GRPA described in Sec. IIII. In the limit $\mathbf{q} \rightarrow \infty$, the vertex corrections vanish and the response function $\chi \rightarrow \chi^{0}$ where $\chi^{0}$ is the response function evaluated in the HFA. Thus, in this limit, the collective mode frequencies must correspond to transitions between a filled and an empty eigenstate of the Hartree-Fock Hamiltonian. At finite value of q, some modes mix together and it becomes difficult to identify their character (layer, orbital, spin transitions, etc.). Our numerical results are summarized in Fig. 9 for the coherent phases and in Fig. 10 for the incoherent phases.
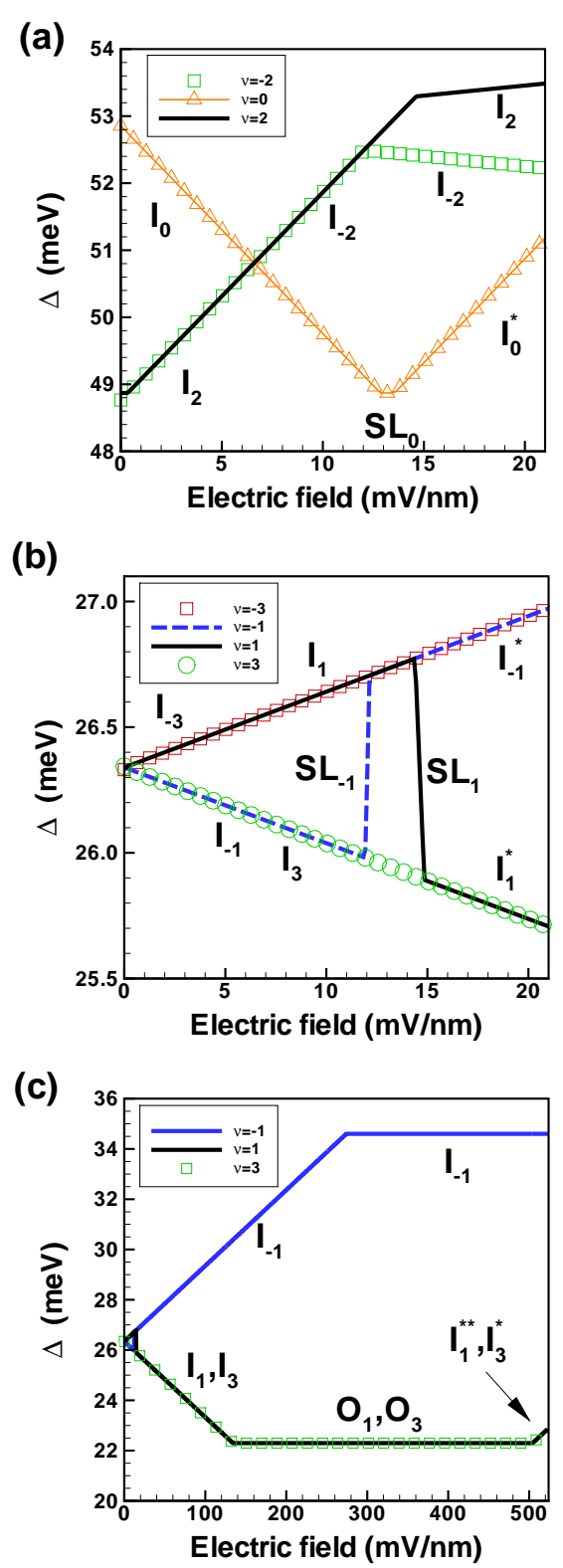

FIG. 8: (Color online) Variation of the transport gap with the perpendicular electric field in the different phases of the C2DEG in Landau level $N=0$. The kink in the behavior of $I_{ \pm 2}$ are due to level crossings. A value of $\kappa=5$ is assumed for the dielectric constant and $B=10 \mathrm{~T}$.

\section{Goldstone modes}

The coherent phases sustain one gapless (Goldstone) mode. The number of Goldstone modes is indicated for each phase in Fig. 5. For example, in phase $L_{-3}$, this mode is due to the fact that the layer pseudospin $\mathbf{L}$ can rotate freely around the $\widehat{\mathbf{z}}$ axis. The same situation occurs for the coherent phase $O_{\nu}$ where again the orbital pseudospin $\mathbf{O}$ can rotate freely around the $\widehat{\mathbf{z}}$ axis. Phases $L_{ \pm 2}$ support coherence in both $n=0$ and $n=1$ orbitals and we can define a layer pseudospin $\mathbf{L}_{0}$ for $n=0$ and $\mathbf{L}_{1}$ 

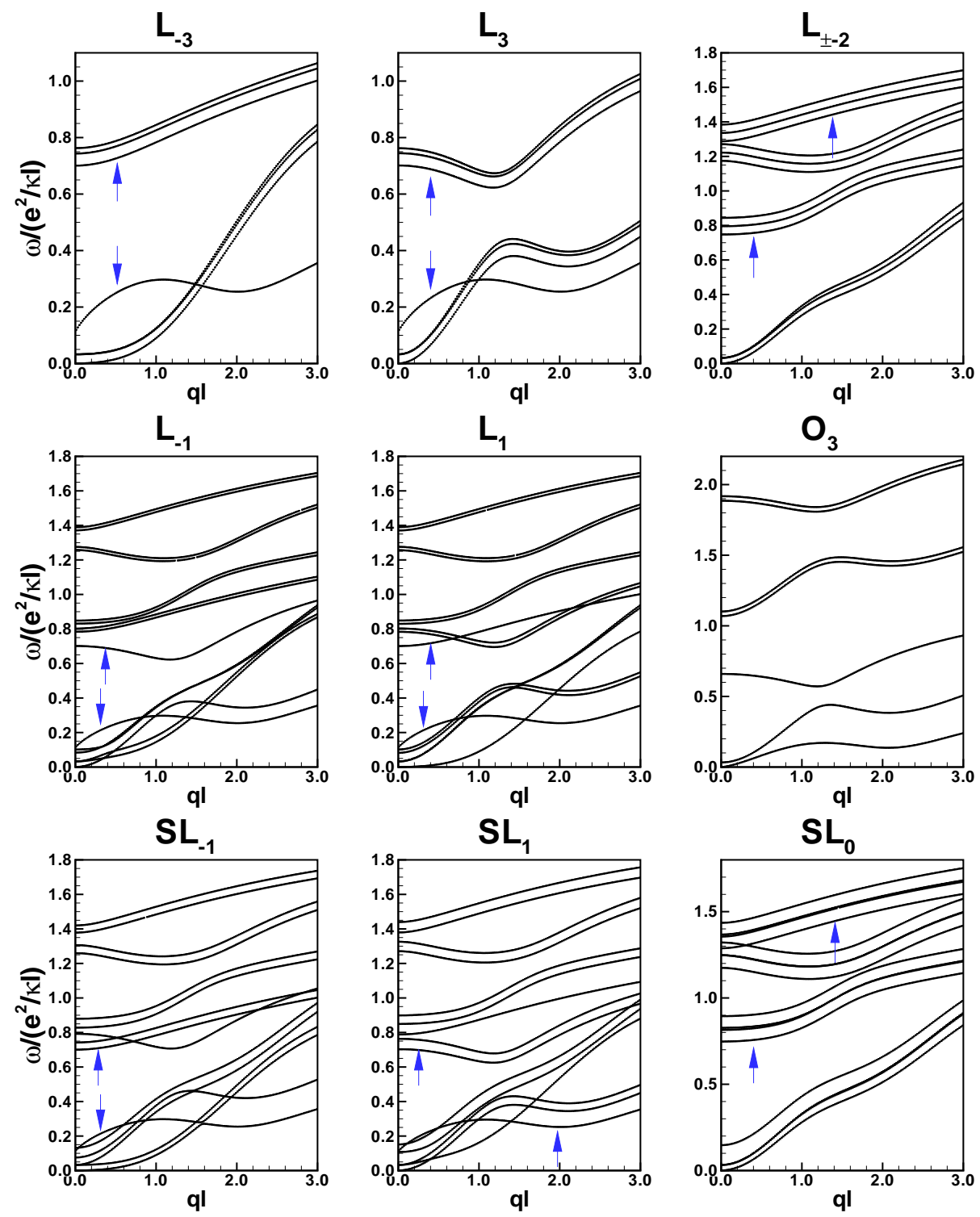

FIG. 9: (Color online) Dispersion of the collective modes in the coherent phases. The (blue) arrows point to the modes which are active in optical absorption experiments. All modes are evaluated at $B=10 \mathrm{~T}$ with $\kappa=5$. The bias in units of $e^{2} / \kappa \ell=35.6$ $\mathrm{meV}$ (corresponding to a frequency $\nu=e^{2} / h \kappa \ell=8.6 \times 10^{12} \mathrm{~Hz}$ ) is respectively: $\Delta_{B}=0.0005$ for $L_{-3}, L_{1} ; \Delta_{B}=0.001$ for $L_{3}, L_{-1} ; \Delta_{B}=0.002$ for $L_{-2} ; \Delta_{B}=1.26$ for $O_{3} ; \Delta_{B}=1.115$ for $S L_{-1} ; \Delta_{B}=0.14$ for $S L_{1}$ and $\Delta_{B}=0.126$ for $S L_{0}$. In $S L_{0}$ the middle line in each group of three dispersive curves contains two modes which are very close in energy.

for $n=1$. The Goldstone mode in this case correspond to an in-phase rotation of both pseudospins. Alternatively, we can see this mode as a fluctuation of the relative phase of the two order parameters $\left\langle\rho_{1,5}\right\rangle$ and $\left\langle\rho_{3,7}\right\rangle$ in $L_{-2}$ or $\left\langle\rho_{2,6}\right\rangle$ and $\left\langle\rho_{4,8}\right\rangle$ in $L_{2}$.

The dispersion off all gapless modes (with the exception of the gapless mode in the orbital phase) is linear in wave vector at very small wave vector i.e. for $q \ell \lesssim d / \ell$. Phases $L_{ \pm 2}$ have the same collective mode spectrum. The dispersion of the Goldstone mode in phases where the coherence occurs in the orbital $n=1$ (i.e. $L_{-1}, L_{3}, S L_{1}$ ) has a roton minimum while there is none if the coherence occurs in $n=0$ (i.e. $L_{-3}, L_{1}, S L_{-1}$ ). This is due to the particular form factor for $n=1$ involved in the Coulomb matrix elements (see Eqs. (44 47)). Phases $L_{-2}, L_{2}, S L_{0}$ contain coherence in both $n=0$ and $n=1$ and a small shoulder appears in the dispersion.

For phase $L_{\nu}$, the Goldstone mode is the famous layerpseudospin-wave mode which has been extensively studied in semiconductor bilayer ${ }^{35}$ at filling factor $\nu=1$ and detected experimentally $\underline{36}$. In semiconductor bilayer, this mode becomes soft at a finite wave vector as the separation between the layers is increased (around $d / \ell \approx 1$ ). In bilayer graphene $d / \ell<<1$ and the layer-coherent phases are stable. The only instability in the collective modes is seen in the orbital phases $O_{1}$ and $O_{3}$. 
For $\zeta_{1}=0$, the Goldstone mode of $L_{-3}\left(\right.$ and $\left.L_{1}\right)$ has a quadratic dispersion at zero bias and becomes unstable 24 at finite bias. (We have checked that these conclusions remain valid if $\zeta_{1}$ is finite but small.) A consequence of this instability is that the C2DEG is expected to go from a smectic (non-homogeneous) phase at low temperature to an isotropic phase at higher temperature. The smectic phase would lead to anisotropic electrical transport.

The dispersion of the gapless orbital pseudospin-wave mode was studied in detail for the uniform phase ${ }^{25}$ as well as for the crystal and helical phases ${ }^{26}$. In the uniform phase, it has a strongly anisotropic dispersion: linear in the direction of the orbital pseudospins and quadratic in the other directions i.e.

$$
\begin{aligned}
& \omega(\mathbf{q})=\sqrt{2\left(\beta \Delta_{B}-\zeta_{1}\right) q \ell}\left|\sin \left(\theta_{\mathbf{q}}\right)\right| \\
& \omega(\mathbf{q})=\frac{1}{4} \sqrt{\sqrt{2 \pi}\left(\beta \Delta_{B}-\zeta_{1}\right)} q \ell,\left(\theta_{\mathbf{q}}=0, \pi\right),
\end{aligned}
$$

where $\theta_{\mathbf{q}}$ is the angle between the wave vector and the $x$ axis. The Goldstone mode softens at a finite wave vector $q \ell \approx 2$ in the direction perpendicular to the orbital pseudospins at a bias $\Delta_{B}=58.8 \mathrm{meV}(E=173 \mathrm{mV} / \mathrm{nm})$. This suggests a transition to a charge-density-wave state. As we explained above, this transition is preempted by a transition to a crystal phase at $\Delta_{B}=47.7 \mathrm{meV}$.

\section{Spin-wave modes}

The number of spin-wave modes gapped at $\Delta_{Z}$ is indicated for each phase in Fig. 5.

All coherent phases with the exception of $O_{1}, O_{3}$ have two modes gapped at the Zeeman energy $\Delta_{Z}$ at $\mathbf{q}=0$. Their degeneracy is lifted at finite wave vector. Because of the occupation of the levels in $O_{1}$ and $O_{3}$, only one intralayer spin-flip transition is possible in theses phases.

The incoherent phases can have 0,1 or 2 modes gapped at $\Delta_{Z}$. To be gapped at $\Delta_{Z}$, these modes must involve transitions within the same valley and orbitals. From Fig. 5, it is easy to see that no intralayer and intraorbital spin-flip transition is possible for $I_{0}^{*}$ and $I_{ \pm 2}^{*}$ and that the occupation of the levels permit only one such mode in $I_{ \pm 3}, I_{ \pm 1}^{*}$. In $I_{ \pm 2}$, two transitions seem possible but they are degenerate and the coupling between them leaves one mode gapped at $\Delta_{Z}$ and the second mode has its frequency renormalized. The same mechanism operates in phases $I_{ \pm 1}, I_{0}$ resulting in two modes gapped at $\Delta_{Z}$.

\section{Optical absorption}

In the absence of Coulomb interaction, the dynamical conductivity has intra-octet peaks at the bare gap energy $\Delta_{\xi}^{0}=E_{\xi, 1}^{0}-E_{\xi, 0}^{0}=\zeta_{1}+\xi \beta \Delta_{B}$ in addition to the inter-Landau-level peaks which do not appear in our calculation. Fig. 11]shows the absorption in different phases when Coulomb interaction is considered. The number of absorption peaks is also indicated for each phase in Fig. 5 and, in Figs. 910, we identify by (blue) arrows the modes that lead to optical absorption.

In the incoherent phases, optical absorption is possible only for transitions that occur between states with the same valley and spin indices but different orbital indices. The incoherent phases have one or zero absorption peak. The latter case occurs when both levels $n=0,1$ with the same layer and spin indices are filled (phases $I_{ \pm 2}, I_{0}, I_{0}^{*}$ ).

When level $n=0$ is filled, the absorption, as shown in Sec. III.g is exactly at $\omega=\left(\zeta_{1}+\xi \beta \Delta_{B}\right) / \hbar$. When level $n=1$ is filled, the absorption is at $\omega=$ $\left(\zeta_{1}-\beta \Delta_{B}+\Delta_{C} / 4\right) / \hbar$ i.e. affected by exchange corrections. The former case applies to most of the incoherent phases in our phase diagram. The latter case applies to phases $I_{1}^{* *}, I_{2}^{*}$ and $I_{3}^{*}$ which occur at very high bias and are outside the limits of validity of our two-band model. In those phases, level $n=1$ is filled because it is below $n=0$ in energy. In phases $I_{ \pm 2}^{*}$, the two allowed transitions have the same energy and the intensity of the absorption peak is doubled.

Fig. 11] (a) shows the absorption as a function of frequency in phase $I_{-3}$ at finite bias. The absorption is concentrated in one strong peak at the frequency

$$
\begin{aligned}
\nu & =\left(\zeta_{1}+\xi \beta \Delta_{B}\right) / h \\
& =\left(9.8+7.6 \xi \Delta_{B}\left[\text { in } \frac{e^{2}}{\kappa \ell}\right]\right) \times 10^{11} \mathrm{~Hz}
\end{aligned}
$$

at $B=10 \mathrm{~T}$ and $\kappa=5$. The absorption frequency varies widely with bias. For example, at the onset of the transition from $I_{1}^{*}$ to $O_{1}$, the frequency $\nu \rightarrow 0$ (see Eq. (105) ) while $\nu \approx 9.8 \times 10^{11} \mathrm{~Hz}$ at the onset of the $I_{1}$ phase. By contrast, if the absorption occurs in layer $K_{+}$, the minimum frequency is $\nu=9.8 \times 10^{11} \mathrm{~Hz}$ since the absorption frequency must increase with bias in this case.

In the $O_{1}, O_{3}$ phases, the Goldstone mode has orbital character (i.e. electric dipole fluctuations) but does not lead to absorption at finite frequency.

Interestingly, the coherent phases $L_{\nu}$ and $S L_{\nu}$ show two absorption peaks (see Fig. 11). The second peak in $L_{ \pm 1}, L_{ \pm 3}$ is extremely weak however and is absent at zero bias. The two weak peaks in $L_{ \pm 2}$ are also extremely weak and disappear at zero bias. In $L_{-3}, L_{1}$, the first absorption peak at zero bias is exactly at $\hbar \omega=\zeta_{1}$ while for $L_{-1}, L_{3}$, the frequency is at $\hbar \omega=$ $\zeta_{1}+\left(X_{0,0,0,0}^{+,+}-X_{0,0,0,0}^{+,-}-X_{1,1,1,1}^{+,+}+X_{1,1,1,1}^{+,-}\right) / 2$ which is slightly shifted from $\hbar \omega=\zeta_{1}$.

The layer eigenstates of the Hartree-Fock Hamiltonian at zero bias are the symmetric $(S)$ and antisymmetric $(A S)$ combinations of $K_{+}$and $K_{-}$. Using Eqs. (90) and (62), we can show from the GRPA equations that the response functions that enter in Eq. (81) for the absorption in the absence of orbital coherence and at zero bias are of the form $\chi_{0,1,1,0}^{\left(a_{\xi}, \sigma_{1}\right),\left(a_{\xi}, \sigma_{1}\right),\left(a_{\xi}, \sigma_{1}\right),\left(a_{\xi}, \sigma_{1}\right)}(\omega)$ or $\chi_{1,0,01}^{\left(a_{\xi}, \sigma_{1}\right),\left(a_{\xi}, \sigma_{1}\right),\left(a_{\xi}, \sigma_{1}\right),\left(a_{\xi}, \sigma_{1}\right)}(\omega)$ where $a_{\xi}$ now stands for the $S$ or $A S$ layer combinations. That is, the layer 

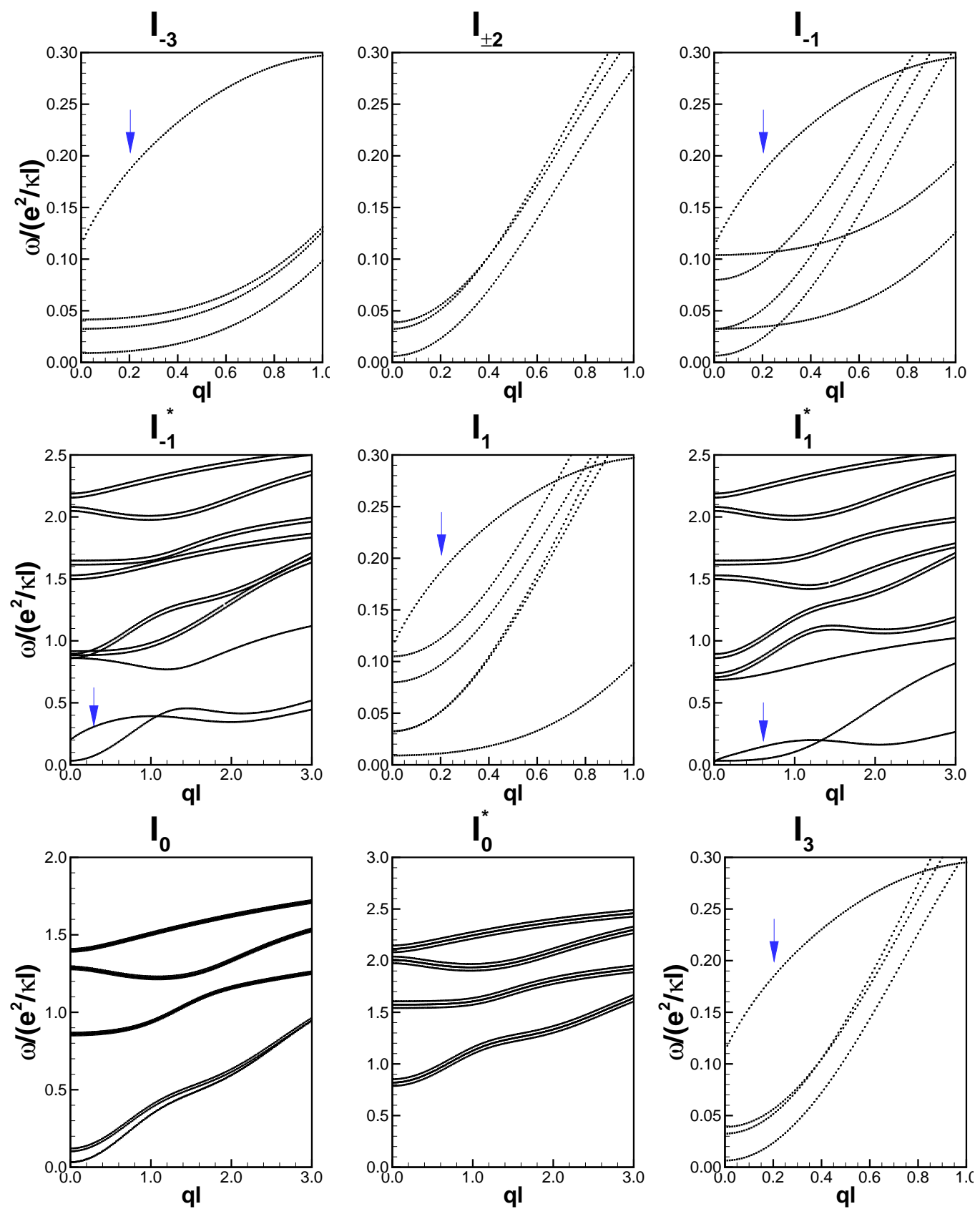

FIG. 10: (Color online) Dispersion of the collective modes in the incoherent phases for $B=10 \mathrm{~T}$ and $\kappa=5$. The bias is in units of $e^{2} / \kappa \ell=35.6 \mathrm{meV}$ corresponding to a frequency $\nu=e^{2} / h \kappa \ell=8.6 \times 10^{12} \mathrm{~Hz}$. The (blue) arrows point to the modes which are active in optical absorption experiments. The bias in units of $e^{2} / \kappa \ell$ is respectively: $\Delta_{B}=0.01$ for $I_{0}, I_{ \pm 1}, I_{ \pm 2}, I_{ \pm 3}$ and $\Delta_{B}=1.0$ for $I_{0}^{*}, I_{ \pm 1}^{*}$. For $I_{ \pm 3}, I_{ \pm 2}, I_{ \pm 1}$ only the first four, three, and six modes respectively are shown. For $I_{0}$, each of the four branches contains four modes which are close in energy. In $I_{0}^{*}$ the middle line in each group of three curves contains two modes.

combination is conserved in the absorption at zero bias and in the absence of orbital coherence. It follows that, in phase $L_{-3}$, the state $|S,+, 0\rangle$ is filled and only the transition $|S,+, 0\rangle \rightarrow|S,+, 1\rangle$ is optically active. For $L_{-2}$, no transition conserving the valley index $(S$ or $A S$ ) is possible and for $L_{-1}$ the only allowed transition is between $|A S,+, 0\rangle \rightarrow|A S,+, 1\rangle$ since levels
$|S,+, 0\rangle,|S,+, 1\rangle,|A S,+, 0\rangle$ are filled. The same argument applies to the spin down states.

At finite but small bias, the $S$ and $A S$ layer combinations are replaced by bonding and anti-bonding combinations i.e. $|B,+, 0\rangle=a\left|K_{-},+, 0\right\rangle+b\left|K_{+},+, 0\right\rangle$ and $|A B,+, 0\rangle=-b\left|K_{-},+, 0\right\rangle+a\left|K_{+},+, 0\right\rangle$ for example where $a$ and $b$ depend on the bias and on the orbital 
index $n$. The strong peak in the absorption corresponds to the transition $|B,+, 0\rangle \rightarrow|B,+, 1\rangle$ for $L_{-3}$ and to $|A B,+, 0\rangle \rightarrow|A B,+, 1\rangle$ for $L_{-1}$. By contrast to the zero bias case, the absorption given by Eq. (79) contains response functions which are not just of the form $\chi_{0,1,1}^{\left(a_{\xi}, \sigma_{1}\right),\left(a_{\xi}, \sigma_{1}\right),\left(a_{\xi}, \sigma_{1}\right),\left(a_{\xi}, \sigma_{1}\right)}(\omega)$ with $a_{\xi}=B, A B$ so that transitions that do not conserve the layer combination $B, A B$ are weakly optically active ${ }^{37}$. A second peak appears in the absorption spectrum which corresponds to the transition $|B,+, 0\rangle \rightarrow|A B,+, 1\rangle$ for $L_{-3}$ and $L_{-1}$. The two weak peaks in $L_{-2}$ come from the transitions $|B,+, 0\rangle \rightarrow|A B,+, 1\rangle$ and $|B,+, 1\rangle \rightarrow|A B,+, 0\rangle$.

Fig. 11 (b) shows the absorption in phases $S L_{0}$ and $S L_{-1}$. In $S L_{-1}$, we find by analyzing the eigenvectors of the modes involved in the optical absorption that the strong peak corresponds to the transition $|B, \eta, 0\rangle \rightarrow$ $|B, \eta, 1\rangle$ and the weak peak to the transition $|B, \eta, 0\rangle \rightarrow$ $|A B, \eta, 1\rangle$ where $|B, \eta, 0\rangle=a\left|K_{-},+, 0\right\rangle+b\left|K_{+},-, 0\right\rangle$ and $|A B, \eta, 0\rangle=-b\left|K_{-},+, 0\right\rangle+a\left|K_{+},-, 0\right\rangle$ and $a, b$ depend on the bias and on the orbital index $n$. The layer combination here are between two states with opposite spin orientations.

The electromagnetic absorption in the crystal and helical phases are much more complex and was discussed previously ${ }^{26}$. In the helical phase, for example, the absorption depends on the orientation of the polarization of the electromagnetic wave in the $x-y$ plane.

The collective mode dispersions computed here for the intra-Landau level $(N=0)$ transitions should be combined with the dispersion of the inter-Landau level magnetoexcitons computed by Sari and Töke $\underline{\underline{23}}$ and by Shizuya ${ }^{38}$ to get a complete picture of the absorption for the C2DEG in bilayer graphene.

\section{EFFECT OF AN IN-PLANE ELECTRIC FIELD}

In this section, we consider the effect of adding a uniform in-plane electric field to the Hartree-Fock Hamiltonian of Eq. (41). The coupling between the C2DEG and this external electric field is given by

$$
H_{E}=-\mathbf{d} \cdot \mathbf{E}_{\|}
$$

where $\mathbf{d}$ is the total dipole moment of the electron gas given by Eq. (70).

The main effect of $\mathbf{E}_{\|}$is to induce orbital coherence. We consider here the case of $\nu=-1$ but a similar effect occur at other filling factors and will be discussed elsewhere ${ }^{39}$. We set $\mathbf{E}_{\|}=-E_{0} \widehat{\mathbf{x}}$. In the ground state, the electric dipoles are aligned with $\mathbf{E}_{\|}$.

With finite $\mathbf{E}_{\|}$, the $S L_{-1}$ state is replaced by a state with spin, orbital, and layer coherences i.e. $S O L_{-1}$. This is represented by the inset in the top-left corner of Fig.
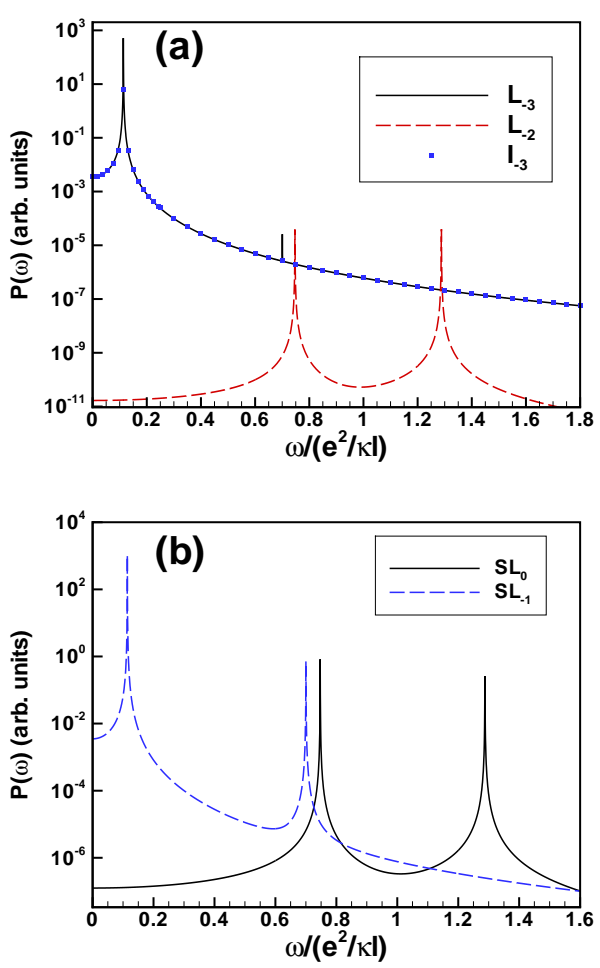

FIG. 11: (Color online) Electromagnetic absorption for $B=$ $10 \mathrm{~T}$ and $\kappa=5$ in phases (a) $L_{-3}\left(\Delta_{B}=0.018 \mathrm{meV}\right), I_{3}$ $\left(\Delta_{B}=0.36 \mathrm{meV}\right)$ and $L_{-2}\left(\Delta_{B}=0.07 \mathrm{meV}\right)$; (b) $S L_{0}\left(\Delta_{B}=\right.$ $4.48 \mathrm{meV})$ and $S L_{-1}\left(\Delta_{B}=4.09 \mathrm{meV}\right)$.

12. The wave function of the ground state becomes

$$
\begin{aligned}
\left|\Psi_{S O L_{-1}}\right\rangle \rightarrow & \prod_{X}\left(a c_{6, X}^{\dagger}+b c_{1, X}^{\dagger}+c c_{3, X}^{\dagger}+d c_{8, X}^{\dagger}(1) 47\right) \\
& \times c_{7, X}^{\dagger} c_{5, X}^{\dagger}|0\rangle
\end{aligned}
$$

with

$$
|a|^{2}+|b|^{2}+|c|^{2}+|d|^{2}=1 .
$$

We show in Fig. 12 how the different polarizations $L_{z}, P_{z}, O_{z}$ and $O_{x}$ change with bias in $S L_{-1}$ when $\mathbf{E}_{\|}$ is increased. The other polarizations are zero. The spin(layer) polarization $S_{z}\left(L_{z}\right)$ increases(decreases) with with $\mathbf{E}_{\|}$until $E_{\|}^{c} \gtrsim 0.2 \mathrm{mV} / \mathrm{nm}$ where it remains constant. The orbital coherence has not yet saturated at $E_{\|}^{c}$. The ground state above this critical electric field is represented in the inset at the top-right corner of Fig. 12 , It is interesting that the spin polarization can be varied in this phase by an external electric field.

The state $\left|\Psi_{S O L_{-1}}\right\rangle$ has one gapless Goldstone mode and its dispersion is anisotropic in wave-vector space (as is the dispersion of the other modes). By contrast, if we apply $\mathbf{E}_{\|}$to a phase $O_{\nu}$, the $\mathrm{U}(1)$ symmetry of the dipoles in the $x-y$ plane is broken. The orbital pseudospin $\mathbf{O}$ is then forced to align with $\mathbf{E}_{\|}$and the Goldstone mode is gapped $^{20}$. 
The absorption in $\left|\Psi_{S O L_{-1}}\right\rangle$ shows two peaks as in $\left|\Psi_{S L_{-1}}\right\rangle$. The first, low-energy, peak is shown in Fig. 13 for two different orientations of the electromagnetic wave polarization. Clearly, the absorption is anisotropic in phase $S O L_{-1}$ by contrast to all the other uniform states that we studied before.

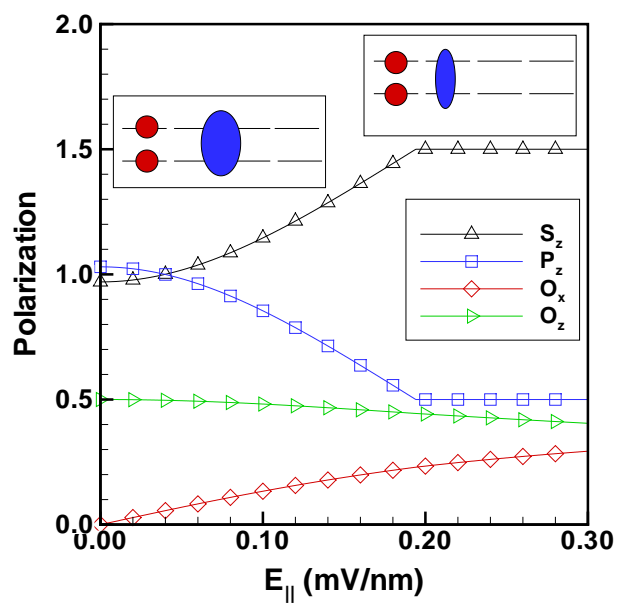

FIG. 12: (Color online) Variation of the spin and layer polarization and of the orbital coherence with an applied in-plane electric field in phase $S O L_{-1}$. The bias $\Delta_{B}$ has been taken near the middle of the $S L_{-1}$ phase where $\left\langle S_{z}\right\rangle=\left\langle P_{z}\right\rangle \approx 1$. Parameters are $B=10 \mathrm{~T}$ and $\kappa=5$.

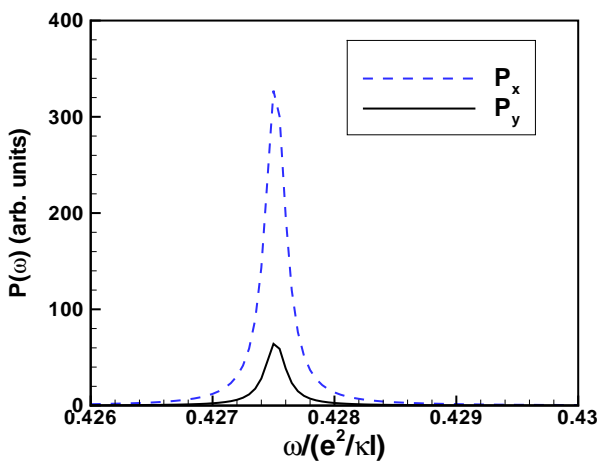

FIG. 13: (Color online) Electromagnetic absorption in the $S O L_{-1}$ phase for two different polarizations of the electromagnetic field.

\section{CONCLUSION}

In this work, we have derived the phase diagram of the C2DEG in a Bernal-stacked graphene bilayer. For the non-interacting Hamiltonian, we used a tight-binding model with the hopping terms $\gamma_{0}, \gamma_{1}, \gamma_{4}$ and $\delta$ and introduced a potential bias between the two layers given by
$\Delta_{B}$. The Coulomb interaction was treated in the HartreeFock approximation. To reduce the complexity of the problem, we used an effective two-band model which described the low-energy behavior of the C2DEG and is valid for $\Delta_{B}<<\gamma_{1}$. We also restricted the Hilbert space to the $N=0$ Landau level only and worked at zero temperature. Our method allows us to include both coherent and incoherent phases in the phase diagram. Indeed, we found phases with layer coherence at small bias, spin and layer coherence at intermediate bias and orbital coherence at large bias. The application of a parallel electric field, as we showed, can also lead to a state with orbital, layer and spin coherence.

We have included in our analysis the hopping parameter $\gamma_{4}$ which is often neglected in theoretical calculations. In our calculations, we find that the phase diagram in sensitive to the precise value of this parameter. If the value of this parameter is modified, the phases that we have discussed are still present in the phase diagram but they occur at different bias. Moreover, other phases may appear. For example, with $\gamma_{4}=0$, the orbital-coherent phase becomes possible at $\nu=-1$ and a new phase with valley and orbital coherence appears as discussed in Ref. 25.

We have written down the ground-state wave function for each phase in the global phase diagram of the C2DEG. We have also calculated for each phase the transport gap, the spin polarization, the collective mode dispersions and the electromagnetic absorption spectrum. The change in these properties from one phase to another should facilitate their experimental identification. Strictly speaking, however, our results are only valid within the limits of validity of the approximations listed above. In particular, we have neglected screening corrections which are known to reduce significantly the transport gaps. These corrections were considered for the incoherent phases in Ref. 17. In principle, these screening corrections should be smaller at larger magnetic field. The stability of the different phases that we found should also be studied by considering quantum and thermal fluctuations as well as disorder effects.

More subtle corrections specific to graphene have also been considered by Shizuya $20-22$. The quantum fluctuations of the Dirac sea (the filled Landau levels from the valence band) have been shown to be sizable and to lead to corrections of the energy of the octet of states in $N=0 \underline{19}$. According to Shizuya, the orbital degeneracy of the zero-energy levels is lifted by Coulombic vacuum fluctuations, leading to an appreciable shift and splitting of the $n=0$ and $n=1$ levels and to a negative capacitance effect that blocks the rotation of the valley pseudospins. The negative capacitance effect appears when the full four bands of the tight-binding model are considered.

A more complete calculation would include all these effects and allow a more direct comparison with the experimental results. 


\section{Acknowledgments}

R. Côté was supported by a grant from the Natural Sciences and Engineering Research Council of
Canada (NSERC). Computer time was provided by Calcul Québec and Compute Canada.
1 For a review on bilayer graphene, see Edward McCann and Mikito Koshino, arXiv:1205.6953 Johan Nilsson, A. H. Castro Neto, F. Guinea, and N. M. R. Peres, Phys. Rev. B 78, 045405 (2008) and Ref. 2 below.

2 Eduardo V Castro, K S Novoselov, S V Morozov, N M R Peres, J M B Lopes dos Santos, Johan Nilsson, F. Guinea, A K Geim and A H Castro Neto, J. Phys.: Condens. Matter 22, 175503 (2010).

3 Yafis Barlas, Kun Yang and A. H. MacDonald, Nanotechnology 23, 052001 (2012).

${ }^{4}$ K. S. Novoselov, E. McCann, S. V. Morozov, V. I. Fal'ko, M. I. Katsnelson, U. Zeitler, D. Jiang, F. Schedin, and A. K. Geim, Nat. Phys. 2, 177 (2006)

5 Benjamin E. Feldman, Jens Martin, and Amir Yacoby, Nat. Phys. 5, 889 (2009).

6 J. Martin, B. E. Feldman, R. T. Weitz, M. T. Allen, and A. Yacoby, Phys. Rev. Lett. 105, 256806 (2010).

7 Wenzhong Bao, Zeng Zhao, Hang Zhang, Gang Liu, Philip Kratz, Lei Jing, Jairo Velasco, Jr., Dmitry Smirnov, and Chun Ning Lau, Phys. Rev. Lett. 105, 246601 (2010).

8 C. R. Dean, A. F. Young, I. Meric, C. Lett, L. Wang, S. Sorgenfrei, K. Watanabe, T. Taniguchi, P. Kim, K. L. Shepard and J. Hone, Nature Nanotechnology, 5, 722 (2010).

9 Y. Zhao, P. Cadden-Zimansky, Z. Jiang, and P. Kim, Phys. Rev. Lett. 104, 066801 (2010).

10 J. Velasco Jr, L. Jing, W. Bao, Y. Lee, P. Kratz, V. Aji, M. Bockrath, C. N. Lau, C. Varma, R. Stillwell, D. Smirnov, Fan Zhang, J. Jung and A. H. MacDonald, Nature Nanotechnology, 7, 156 (2012).

11 F. Freitag, J. Trbovic, M. Weiss, and C. Schönenberger, Phys. Rev. Lett. 108, 076602 (2012).

12 R. T. Weitz, M. T. Allen, B. E. Feldman, J. Martin, and A Yacoby, Science 330, 812 (2010).

13 Yafis Barlas, R. Côté, K. Nomura, and A. H. MacDonald, Phys. Rev. Lett. 101, 097601 (2008).

14 Seyoung Kim, Kayoung Lee, and E. Tutuc, Phys. Rev. Lett. 107, 016803 (2011).

15 Rahul Nandkishore and Leonid Levitov, Phys. Rev. B 82, 115124 (2010); Rahul Nandkishore and Leonid Levitov, Phys. Scr. T146, 014011 (2012).

16 E. V. Gorbar, V. P. Gusynin, and V. A. Miransky, Phys. Rev. B 81, 155451 (2010); id., JETP Letters, 91, 314 (2010); E. V. Gorbar, V. P. Gusynin, V. A. Miransky, and I. A. Shovkovy, Phys. Rev. B 85, 235460 (2012).

17 E. V. Gorbar, V. P. Gusynin, Junji Jia, and V. A. Miransky, Phys. Rev. B 84, 235449 (2011).

18 E. V. Gorbar, V. P. Gusynin, A. B. Kuzmenko, and S. G.
Sharapov, Phys. Rev. B 86, 075414 (2012).

19 K. Shizuya, Phys. Rev. B 86, 045431 (2012).

20 K. Shizuya, Phys. Rev. B 79, 165402 (2009).

21 K. Shizuya, Phys. Rev. B 84, 075409 (2011).

22 T. Misumi and K. Shizuya, Phys. Rev. B 77, 195423 (2008).

23 Judit Sari and Csaba Töke, unpublished (arXiv:1209.0638).

24 Yafis Barlas, R. Côté, J. Lambert, and A. H. MacDonald, Phys. Rev. Lett. 104, 096802 (2010).

25 R. Côté, Jules Lambert, Yafis Barlas, and A. H. MacDonald, Phys. Rev. B 82, 035445 (2010).

${ }^{26}$ R. Côté, J. P. Fouquet, and Wenchen Luo, Phys. Rev. B 84, 235301 (2011).

27 Edward McCann and Vladimir I. Fal'ko, Phys. Rev. Lett. 96, 086805 (2006).

28 Fan Zhang, Bhagawan Sahu, Hongki Min, and A. H. MacDonald, Phys. Rev. B 82, 035409 (2010).

29 B. Partoens and F. M. Peeters, Phys. Rev. B 74, 075404 (2006).

30 M. O. Goerbig, R. Moessner, and B. Douçot, Phys. Rev. B 74, 161407(R) (2006).

31 For a review on coherent states in semiconductor bilayers, see Z. F. Ezawa, Quantum Hall Effects (World Scientific, Singapore, 2000).

${ }^{32}$ With $\zeta_{1} \neq 0$, the beginning of the orbital phase is at $\Delta_{B}=$ $\zeta_{1} / \beta$ and not at $\Delta_{B, \text { onset }}+\zeta_{1} / \beta$ as erroneously mentionned on page 8 of Ref. 26 .

33 Jung Hoon Han, Jiadong Zang, Zhihua Yang, Jin-Hong Park, and Naoto Nagaosa, Phy. Rev. B 82, 094429 (2010).

34 I. Dzyaloshinsky, J. Phys. Chem. Solids 4, 241 (1958); T. Moriya, Phys. Rev. 120, 91 (1960).

35 H. A. Fertig, Phys. Rev. B 40, 1087 (1989); A. H. MacDonald, P. M. Platzman and G. S. Boebinger, Phys. Rev. Lett. 65, 775 (1990); R. Côté, L. Brey, and A.H. MacDonald, Phys. Rev. B 46, 10239 (1992); Xiao-Gang Wen and A. Zee, Phys. Rev. Lett. 69, 1811 (1992); J. P. Eisenstein and A. H. MacDonald, Nature (London) 432, 691 (2004).

36 I. B. Spielman, J. P. Eisenstein, L. N. Pfeiffer, and K. W. West, Phys. Rev. Lett. 87, 036803 (2001).

37 We remark that the transitions that do not conserve the layer combinations $B, A B$ are absent of the spectrum if the small term $\beta \Delta_{B}$ is artificially set to zero in $\Delta_{a}^{0}, \Delta_{b}^{0}$ in Eq. (79).

38 K. Shizuya, Phys. Rev. B 81, 075407 (2010).

39 J. Lambert and R. Côté, unpublished. 